Journal of Zhejiang University-SCIENCE A (Applied Physics \& Engineering)

ISSN 1673-565X (Print); ISSN 1862-1775 (Online)

www.zju.edu.cn/jzus; www.springerlink.com

E-mail: jzus@zju.edu.cn

\title{
Review:
}

\section{Mechanics of dielectric elastomers: materials, structures, and devices*}

\author{
Feng-bo ZHU ${ }^{1,2}$, Chun-li ZHANG ${ }^{1,2,3}$, Jin QIAN $\$ 1,2,3$, Wei-qiu CHEN ${ }^{1,2,3}$ \\ ('Department of Engineering Mechanics, Zhejiang University, Hangzhou 310027, China) \\ ( ${ }^{2}$ Soft Matter Research Center, Zhejiang University, Hangzhou 310027, China) \\ ( ${ }^{3}$ Key Laboratory of Soft Machines and Smart Devices of Zhejiang Province, Zhejiang University, Hangzhou 310027, China) \\ ${ }^{\dagger}$ E-mail: jqian@zju.edu.cn
}

Received Apr. 15, 2015; Revision accepted Sept. 24, 2015; Crosschecked Oct. 12, 2015

\begin{abstract}
Dielectric elastomers (DEs) respond to applied electric voltage with a surprisingly large deformation, showing a promising capability to generate actuation in mimicking natural muscles. A theoretical foundation of the mechanics of DEs is of crucial importance in designing DE-based structures and devices. In this review, we survey some recent theoretical and numerical efforts in exploring several aspects of electroactive materials, with emphases on the governing equations of electromechanical coupling, constitutive laws, viscoelastic behaviors, electromechanical instability as well as actuation applications. An overview of analytical models is provided based on the representative approach of non-equilibrium thermodynamics, with computational analyses being required in more generalized situations such as irregular shape, complex configuration, and time-dependent deformation. Theoretical efforts have been devoted to enhancing the working limits of DE actuators by avoiding electromechanical instability as well as electric breakdown, and pre-strains are shown to effectively avoid the two failure modes. These studies lay a solid foundation to facilitate the use of DE materials, structures, and devices in a wide range of applications such as biomedical devices, adaptive systems, robotics, energy harvesting, etc.
\end{abstract}

Key words: Artificial muscle, Smart material, Dielectric elastomer (DE), Electromechanical coupling, Constitutive law, Viscoelasticity, Electromechanical instability, Actuation

http://dx.doi.org/10.1631/jzus.A1500125

CLC number: O33; O39

\section{Introduction}

Artificial muscles, a term for man-made materials, structures or devices that reversibly contract, expand, bend or twist in response to external stimuli, have attracted great attention and have advanced rapidly in the last two decades. Research on artificial muscles often involves people from a diversity of disciplines, with an ultimate goal of

\footnotetext{
* Corresponding author

* Project supported by the National Natural Science Foundation of China (No. 11321202), and the Zhejiang Provincial Natural Science Foundation of China (No. LR16A020001)

(D) ORCID: Jin QIAN, http://orcid.org/0000-0002-3597-5460

(C) Zhejiang University and Springer-Verlag Berlin Heidelberg 2016
}

surpassing natural muscles and exploring applications in biomedical devices, adaptive systems, robotics, energy harvesting, etc. Various types of smart materials have been used as artificial muscles with assumed response to external stimuli, including dielectric elastomers (DEs), ferroelectric polymers (FPs), ionic polymer-metal composites (IPMCs), and shape-memory polymers (SMPs). Among those listed, DEs that are usually made of electroactive polymers can readily transform electric energy into mechanical work, enabling them to mimic the function and performance of natural muscles (Shankar et al., 2007; Brochu and Pei, 2010; Kornbluh et al., 2012; Suo, 2012; Zhao and Wang, 2014). Specific examples of DEs include acrylics, silicones, polyurethanes, fluoroelastomers, and ethylene-propylene rubbers. Key 
attributes of such materials, including large actuation strain, fast response, light weight, silent operation, and low cost, provide the unique combination for the task of muscle-like actuation and thus gain increasing attention for a diversity of structures, devices, and applications over recent years (O'Halloran et al., 2008; Carpi et al., 2010; Anderson et al., 2012; Sheng et al., 2012; Graf et al., 2014).

Large deformation is crucial for requisite functions in muscle-like actuation. DE actuators can produce surprisingly large deformations in response to externally applied electric fields. A prototype of a DE actuator appears as a planar and thin membrane sandwiched between two compliant and oppositely charged electrodes, which is essentially a capacitor as shown in Fig. 1a (Suo, 2010; Zhao et al., 2011; Li B. et al., 2014). Once an electric field is applied across the electrodes, the charges of opposite sign cause Coulombic attraction that compresses the material in the thickness direction and expands it in the membrane plane, with a relatively large strain magnitude and high strain rate. The shape of the elastomer is recovered if the voltage is removed. Nowadays, the typical operating voltage for DE functioning falls into the range $500 \mathrm{~V}$ to $10 \mathrm{kV}$, and the areal strain can be as much as $1000 \%$ (Wissler and Mazza, 2005; Zhang et al., 2005; Li T.F. et al., 2013).

Early electroactive polymers were shown to produce relatively small strains, while more advanced DEs that are capable of large deformation over $100 \%$ have emerged since the beginning of the 1990s. This trend of an increasing deformation regime in DE applications calls for a paradigm shift in modeling the electromechanical coupling of DE behaviors with critical issues such as instability and failure. It is therefore imperative to develop quantitative approaches to how these materials, structures, and devices behave in response to various forms of electric excitation as well as the mechanical load/constraint in the designing, manufacturing, and functioning stage of DE products.

The functioning, capability, and reliability of actuators made of DEs have been theoretically and computationally modeled in different configurations, such as cylindrical (Carpi and de Rossi, 2004), spherical (Mockensturm and Goulbourne, 2006), pre-strained circular (Wissler and Mazza, 2005; Chen and Dai, 2012), and so on, in which particular forms of strain energy function have been shown to ade- quately represent the material constitutions and describe their actuation behaviors.

In experiments, the electromechanical response of some DEs has been exhibited to be highly ratedependent and hysteretic, implying significant viscoelasticity and convoluted time scales in the materials. Moreover, the actuation strain of DEs is strongly limited by several failure modes such as electromechanical instability and electric breakdown. To avoid these vital issues and achieve a sufficiently large deformation under electric stimuli, stability and hysteresis analyses have also been performed with results showing that the electromechanical stability of DE actuators can be tuned by the magnitude and distribution of pre-stress (Zhao and Suo, 2007; Zhao et al., 2007). This paper aims to provide an overview of recent theoretical and numerical studies that outline the existing approaches and methods in mechanical analyses of DEs, and is organized as follows. We first introduce the basic theory of the working principles of DEs and summarize the equation system governing the key physical quantities in the analyses. Then, we discuss the prevailing approach of constructing the constitutive laws of such compliant and electroactive materials with or without the effects of viscoelasticity (Sheng et al., 2012). One of the main failure modes, i.e., the electromechanical instability of DEs, is demonstrated, with results showing that pre-stretch is an effective strategy to markedly enhance DE deformation without causing instability. A number of representative applications of DE actuators are summarized in Section 5. The outlook for future research opportunities in studying the mechanics of DEs is provided in the final part.

\section{Working principles of DE actuators}

\subsection{Basic theory of DEs}

DEs are soft materials that constantly undergo large and reversible deformation in response to electric fields. An external electric field can easily cause a DE to stretch several times its original dimension. Artificial muscles made of DEs generally require two independent variations (i.e., electrical and mechanical) in any theoretical description. In the following, only isothermal processes are considered for the purpose of demonstration, and temperature effects are beyond the scope of this review (Suo, 2010). It should be mentioned that the following derivations and analyses 
to describe the interaction between mechanical deformation and electric fields in DEs are not unique, and other theoretical frameworks exist and equivalently capture the electroelastic coupling in DEs. For example, Dorfmann and Ogden (2014) have developed a general theoretical framework, in the form of tensors, which applies for a broad range of electroactive and magnetoactive materials. In the following, we adopt the treatments more specific to DEs as being more consistent with the prevailing discussion on the topic.

Consider a planar and thin membrane of DE in its reference state, free of voltage and force, as shown in Fig. 1a. The original dimensions of the DE membrane are $L_{1}, L_{2}$, and $L_{3}$. In the current state, the DE membrane is subjected to forces $P_{1}, P_{2}$, and $P_{3}$, with the two electrodes set to a prescribed voltage $\phi$. Upon application of the forces and voltage, the new dimensions of the membrane become $l_{1}, l_{2}$, and $l_{3}$, with electric charges $\pm Q$ accumulated on the opposing electrodes (Fig. 1b). Therefore, the elastomer is stretched by $\lambda_{1}=l_{1} / L_{1}, \lambda_{2}=l_{2} / L_{2}$, and $\lambda_{3}=l_{3} / L_{3}$.

The thermodynamic behaviors of the DE membrane can be characterized by the Helmholtz free energy, denoted as $F$. As the dimensions of the membrane change incrementally by $\delta l_{1}, \delta l_{2}$, and $\delta l_{3}$, the mechanical work done by the forces is $P_{1} \delta l_{1}+P_{2} \delta l_{2}+P_{3} \delta l_{3}$. Similarly, when a small quantity of electric charge $\delta Q$ flows through the conducting circuit, the work done by the applied voltage is $\phi \delta Q$. The law of energy conservation requires that the net work done by the forces and voltage should be equal to the change in the Helmholtz free energy, namely,

$$
\delta F=P_{1} \delta l_{1}+P_{2} \delta l_{2}+P_{3} \delta l_{3}+\phi \delta Q
$$

It is convenient to define the nominal density of the Helmholtz free energy as $W=F /\left(L_{1} L_{2} L_{3}\right)$, the nominal stresses as $\tilde{\sigma}_{1}=P_{1} /\left(L_{2} L_{3}\right), \quad \tilde{\sigma}_{2}=P_{2} /\left(L_{1} L_{3}\right)$, and $\tilde{\sigma}_{3}=P_{3} /\left(L_{1} L_{2}\right)$, the true stresses as $\sigma_{1}=P_{1} /\left(l_{2} l_{3}\right)$, $\sigma_{2}=P_{2} /\left(l_{1} l_{3}\right)$ and $\sigma_{3}=P_{3} /\left(l_{1} l_{2}\right)$, the nominal electric field as $\tilde{E}=\phi / L_{3}$, the true electric field as $E=\phi / l_{3}$, the nominal electric displacement as $\tilde{D}=Q /\left(L_{1} L_{2}\right)$, and the true electric displacement as $D=Q /\left(l_{1} l_{2}\right)$. The amount of electric charge on each electrode can be related to the nominal electric displacement through $Q=\tilde{D} L_{1} L_{2}$. When the DE membrane is subjected to forces and voltage in its current state, the charge variation is simply $\delta Q=L_{1} L_{2} \delta \tilde{D}$.

(a)

(b)

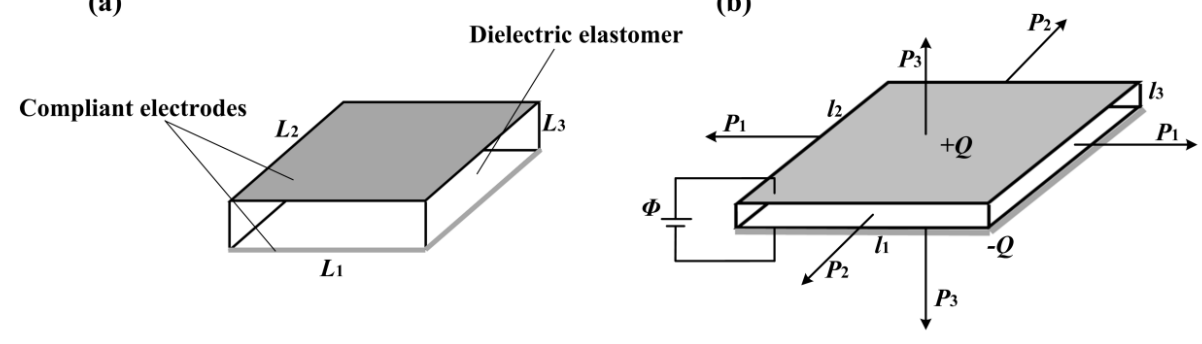

(c)

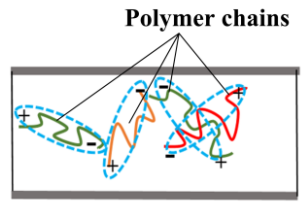

(d)

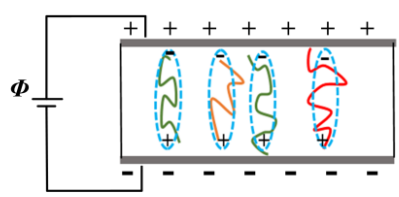

(e)

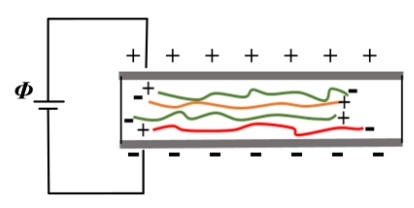

Fig. 1 Working principle of DE actuators

(a) Schematics of a membrane of DE with coated electrodes on both sides; (b) By applying an electric voltage, the electrostatic force compresses the DE membrane in the thickness direction and expands it in the membrane plane. The DE membrane recovers its original configuration when the voltage is removed; (c) An elastomer film at its reference state, with randomly distributed polymer dipoles; (d) Polarization of an ideal DE, in which the polymer dipoles are polarized freely; (e) A non-ideal DE under large deformation. The stress field is perpendicular to the direction of electric field, which impedes the polarization of dipoles (Anderson et al., 2012; Foo et al., 2012a) 
The nominal density of the Helmholtz free energy $W$ generally depends on the mechanical and electric displacement as

$$
W=W\left(\lambda_{1}, \lambda_{2}, \lambda_{3}, \tilde{D}\right)
$$

Dividing both sides of Eq. (1) by $L_{1} L_{2} L_{3}$, we obtain:

$$
\delta W=\tilde{\sigma}_{1} \delta \lambda_{1}+\tilde{\sigma}_{2} \delta \lambda_{2}+\tilde{\sigma}_{3} \delta \lambda_{3}+\tilde{E} \delta \tilde{D}
$$

Inserting Eq. (2) into Eq. (3) leads to

$$
\begin{gathered}
\left(\frac{\partial W}{\partial \lambda_{1}}-\tilde{\sigma}_{1}\right) \delta \lambda_{1}+\left(\frac{\partial W}{\partial \lambda_{2}}-\tilde{\sigma}_{2}\right) \delta \lambda_{2}+\left(\frac{\partial W}{\partial \lambda_{3}}-\tilde{\sigma}_{3}\right) \delta \lambda_{3} \\
+\left(\frac{\partial W}{\partial \tilde{D}}-\tilde{E}\right) \delta \tilde{D}=0 .
\end{gathered}
$$

This condition of conservation should be satisfied for an arbitrary set of small variations $\delta \lambda_{1}, \delta \lambda_{2}, \delta \lambda_{3}$, and $\delta \tilde{D}$. Thus,

$$
\begin{gathered}
\tilde{\sigma}_{1}=\frac{\partial W\left(\lambda_{1}, \lambda_{2}, \lambda_{3}, \tilde{D}\right)}{\partial \lambda_{1}}, \\
\tilde{\sigma}_{2}=\frac{\partial W\left(\lambda_{1}, \lambda_{2}, \lambda_{3}, \tilde{D}\right)}{\partial \lambda_{2}}, \\
\tilde{\sigma}_{3}=\frac{\partial W\left(\lambda_{1}, \lambda_{2}, \lambda_{3}, \tilde{D}\right)}{\partial \lambda_{3}}, \\
\tilde{E}=\frac{\partial W\left(\lambda_{1}, \lambda_{2}, \lambda_{3}, \tilde{D}\right)}{\partial \tilde{D}} .
\end{gathered}
$$

It can be seen that once the form of the free energy function $W\left(\lambda_{1}, \lambda_{2}, \lambda_{3}, \tilde{D}\right)$ is explicitly provided for a particular material, the constitutive equations follow immediately.

The equations of state can also be established in terms of true quantities. As mentioned above, the true electric displacement is defined as $D=Q /\left(l_{1} l_{2}\right)$, and the amount of electric charge on each electrode related to the true electric displacement can be converted to $Q=D l_{1} l_{2}$. When the DE membrane is subjected to external forces and voltage, the variation of electric charge can be expressed as

$$
\delta Q=D l_{2} \delta l_{1}+D l_{1} \delta l_{2}+l_{1} l_{2} \delta D
$$

Again, one can divide both sides of Eq. (1) by $L_{1} L_{2} L_{3}$ in conjunction with Eq. (9), which leads to

$$
\begin{aligned}
\delta W= & \left(\sigma_{1}+D E\right) \lambda_{2} \lambda_{3} \delta \lambda_{1}+\left(\sigma_{2}+D E\right) \lambda_{1} \lambda_{3} \delta \lambda_{2} \\
& +\sigma_{3} \lambda_{1} \lambda_{2} \delta \lambda_{3}+\lambda_{1} \lambda_{2} \lambda_{3} E \delta D .
\end{aligned}
$$

For DE materials that are usually taken to be incompressible, the membrane volume is conserved (i.e., $\left.l_{1} l_{2} l_{3}=L_{1} L_{2} L_{3}\right)$ and therefore

$$
\lambda_{1} \lambda_{2} \lambda_{3}=1 .
$$

It should be noted that in circumstances such as large hydrostatic pressure or specially imposed boundary conditions, the volume change of $\mathrm{DE}$ materials can be significant and material compressibility should be considered, referring to Liu et al. (2011). Returning to the incompressible case with $\lambda_{1}$ and $\lambda_{2}$ as independent variables, $\lambda_{3}=\lambda_{1}^{-1} \lambda_{2}^{-1}$ and $\delta \lambda_{3}=$ $-\lambda_{1}{ }^{-2} \lambda_{2}{ }^{-1} \delta \lambda_{1}-\lambda_{2}{ }^{-2} \lambda_{1}{ }^{-1} \delta \lambda_{2}$. Eq. (10) becomes

$$
\delta W=\frac{\sigma_{1}-\sigma_{3}+D E}{\lambda_{1}} \delta \lambda_{1}+\frac{\sigma_{2}-\sigma_{3}+D E}{\lambda_{2}} \delta \lambda_{2}+E \delta D
$$

The free energy density $W$ reduces to a function of $\lambda_{1}$ and $\lambda_{2}$ as well as the electric displacement $D$, namely $W\left(\lambda_{1}, \lambda_{2}, D\right)$, and Eq. (12) becomes

$$
\begin{aligned}
& \left(\frac{\delta W}{\delta \lambda_{1}}-\frac{\sigma_{1}-\sigma_{3}+D E}{\lambda_{1}}\right) \delta \lambda_{1}+\left(\frac{\delta W}{\delta \lambda_{2}}-\frac{\sigma_{2}-\sigma_{3}+D E}{\lambda_{2}}\right) \delta \lambda_{2} \\
& \quad+\left(\frac{\delta W}{\delta D}-E\right) \delta D=0 .
\end{aligned}
$$

For incompressible DEs, Eq. (13) holds for arbitrary variations of $\delta \lambda_{1}, \delta \lambda_{2}$, and $\delta D$, which is equivalent to three separated equations:

$$
\begin{aligned}
\sigma_{1}-\sigma_{3} & =\lambda_{1} \frac{\partial W\left(\lambda_{1}, \lambda_{2}, D\right)}{\partial \lambda_{1}}-E D, \\
\sigma_{2}-\sigma_{3} & =\lambda_{2} \frac{\partial W\left(\lambda_{1}, \lambda_{2}, D\right)}{\partial \lambda_{2}}-E D, \\
E & =\frac{\partial W\left(\lambda_{1}, \lambda_{2}, D\right)}{\partial D} .
\end{aligned}
$$

The true forces and voltage can be solved as long as the energy density function $W\left(\lambda_{1}, \lambda_{2}, D\right)$ is explicitly provided for an incompressible DE. 


\subsection{Ideal DEs}

Experimental observations have found that the permittivity $\varepsilon$ of some DEs is nearly constant during deformation (Kofod et al., 2001; Zhang et al., 2005). A reasonable explanation of this observation is the following: such elastomers are 3D networks consisting of long and flexible polymer chains, which interconnect via covalent cross-links. Each polymer chain consists of a large number of monomers. In the case that the presence of cross-links has negligible effects on the polarization of monomers, these elastomers can be idealized to polarize as freely as a polymer melt, as illustrated in Figs. 1c and 1d (Davies et al., 2002).

With the idealization, the dielectric behavior of such elastomers is assumed to be well represented by that of a polymer melt. The relation between the true electric displacement and the true electric field is therefore

$$
D=\varepsilon E,
$$

where $\varepsilon$ is the permittivity of the elastomers that is taken to be constant and independent of deformation.

For fixed $\lambda_{1}$ and $\lambda_{2}$, one can integrate Eq. (12) with respect to $D$ and use the relation in Eq. (17), and the result is

$$
W\left(\lambda_{1}, \lambda_{2}, D\right)=W_{\mathrm{s}}\left(\lambda_{1}, \lambda_{2}\right)+\frac{D^{2}}{2 \varepsilon},
$$

where $W_{\mathrm{s}}\left(\lambda_{1}, \lambda_{2}\right)$ is the strain energy density associated with the in-plane stretches of the material, and $D^{2} /(2 \varepsilon)$ terms are the Helmholtz free energy density associated with electric polarization. Note that the condition of incompressibility is implied throughout the analyses. Eq. (18) is known as the model of ideal DEs, in which the contributions of mechanical stretches and electric polarization to the free energy function are separated. Consequently, the electromechanical coupling in such an ideal DE is merely a geometric effect represented by the relation $Q=L_{1} L_{2} \lambda_{1} \lambda_{2} D$.

Combining the expressions in Eqs. (14), (15), (17) and (18) for incompressible and ideal DEs, one obtains

$$
\begin{gathered}
\sigma_{1}-\sigma_{3}=\lambda_{1} \frac{W_{\mathrm{s}}\left(\lambda_{1}, \lambda_{2}\right)}{\partial \lambda_{1}}-\varepsilon E^{2}, \\
\sigma_{2}-\sigma_{3}=\lambda_{2} \frac{W_{\mathrm{s}}\left(\lambda_{1}, \lambda_{2}\right)}{\partial \lambda_{2}}-\varepsilon E^{2} .
\end{gathered}
$$

Once the strain energy density function $W_{\mathrm{s}}\left(\lambda_{1}, \lambda_{2}\right)$ and the permittivity $\varepsilon$ are specified, the equations of state can be easily obtained by Eqs. (19) and (20). These results have been justified in terms of Maxwell stress, and can be interpreted as the predictive model of ideal DEs.

It is of key importance to choose an appropriate form for the strain energy density function $W_{\mathrm{s}}$ in describing the electromechanical responses of DEs. For example, by adopting the neo-Hookean model with a single material parameter, Leng et al. (2009) and Li et al. (2011) studied the electromechanical stability problem of a DE membrane during actuation. Liu et al. (2010) calculated the allowable area of a DE energy harvester based on the Mooney-Rivlin type energy function. In order to describe the electromechanical behaviors of a particular DE material, a key procedure is to build up its constitutive law with a proper energy function. Most of the existing analyses were demonstrated by choosing specific forms of strain energy function, but the approach can be generalized to strain energy functions of other forms for quantitatively different behaviors. Commonly adopted strain energy functions, such as the neoHookean model, Gent model, Mooney-Rivlin model, Yeoh model, and Ogden model, are listed in Table 1. Some of the listed functions have also been employed in describing the nonlinear elastic behaviors of rubber-like materials, as exemplified by Boyce and Arruda (2000).

The Gent model (Gent, 1996) is a commonly used energy function for DEs that consist of long and flexible polymer chains from a microscopic point of view. Under stretching, the end-to-end distance of each polymer chain increases and eventually approaches the contour length that sets a finite stretching limit, and such elastomers usually stiffen significantly as the stretch approaches the limit. To account for the stretching limit, the Gent model assumes that

$$
W_{\mathrm{s}}\left(\lambda_{1}, \lambda_{2}\right)=-\frac{G J_{\lim }}{2} \log \left(1-\frac{\lambda_{1}^{2}+\lambda_{2}^{2}+\lambda_{1}^{-2} \lambda_{2}^{-2}-3}{J_{\lim }}\right)
$$


Table 1 Strain energy density functions adopted in DE modeling

\begin{tabular}{|c|c|c|c|}
\hline Energy function & Function form & Strain & Reference \\
\hline $\begin{array}{l}\text { neo-Hookean } \\
\text { model }\end{array}$ & $W_{\mathrm{s}}=\frac{G}{2}\left(\lambda_{1}^{2}+\lambda_{2}^{2}+\lambda_{1}^{-2} \lambda_{2}^{-2}-3\right)$ & $30 \%-40 \%$ & $\begin{array}{l}\text { (Dollhofer et al., 2004; Zhao and } \\
\text { Suo, 2007; Wang H. et al., } \\
\text { 2013) }\end{array}$ \\
\hline Gent model & $W_{\mathrm{s}}=-\frac{G J_{\lim }}{2} \log \left(1-\frac{\lambda_{1}^{2}+\lambda_{2}^{2}+\lambda_{1}^{-2} \lambda_{2}^{-2}-3}{J_{\lim }}\right)$ & $30 \%-155 \%$ & $\begin{array}{l}\text { (Huang and Suo, 2012; Lu et al., } \\
\text { 2012; Qu et al., 2012; Zhou et } \\
\text { al., 2013; 2014a) }\end{array}$ \\
\hline $\begin{array}{l}\text { Mooney-Rivlin } \\
\text { model }\end{array}$ & $\begin{array}{l}W_{\mathrm{s}}=C_{1}\left(I_{1}-3\right)+C_{2}\left(I_{2}-3\right), \\
I_{1}=\lambda_{1}^{2}+\lambda_{2}^{2}+\lambda_{3}^{2}, I_{2}=\lambda_{1}^{2} \lambda_{2}^{2}+\lambda_{2}^{2} \lambda_{3}^{2}+\lambda_{3}^{2} \lambda_{1}^{2}\end{array}$ & $<200 \%$ & (Liu et al., 2010) \\
\hline Yeoh model & $\begin{array}{l}W_{\mathrm{s}}=\frac{C_{1}}{2}\left(I_{1}-3\right)+\frac{C_{2}}{2}\left(I_{1}-3\right)^{2}+\frac{C_{3}}{2}\left(I_{1}-3\right)^{3}, \\
I_{1}=\lambda_{1}^{2}+\lambda_{2}^{2}+\lambda_{3}^{2}\end{array}$ & $>200 \%$ & (Wissler and Mazza, 2005) \\
\hline Ogden model & $W_{\mathrm{s}}=\sum_{p=1}^{N} \frac{\mu_{p}}{\alpha_{p}}\left(\lambda_{1}^{\alpha_{p}}+\lambda_{2}^{\alpha_{p}}+\lambda_{1}^{-\alpha_{p}} \lambda_{2}^{-\alpha_{p}}-3\right)$ & $100 \%-400 \%$ & $\begin{array}{l}\text { (Coulbourne, 2011; Proulx et al., } \\
\text { 2011; Qu and Suo, 2012) }\end{array}$ \\
\hline
\end{tabular}

where $G$ is the basal shear modulus, and $J_{\lim }$ is a dimensionless parameter representing the extension limit of the polymer chains. Huang and Suo (2012) used this description in modeling an ideal DE membrane and constructed a diagram for the electromechanical phase transition, with the prediction that a specific critical transition point would occur.

\subsection{Non-ideal DEs}

Many studies used the constitutive models of ideal DEs that take the permittivity to be constant, as those mentioned in the preceding section for an isothermal process. However, soft machines made of DE materials, structures, and devices may work in more complicated conditions where the dielectric constant may change. For example, a sufficiently large magnitude of mechanical strain may orient the dominating chain axes of elastomers, restrict the rotation of dipoles and eventually change the material permittivity $\varepsilon$, as illustrated in Fig. 1e. It was indeed found that $\varepsilon$ can be either linear or nonlinear functions, depending on the magnitude of deformation (He et al., 2009; Leng et al., 2009). Some other studies also showed that the environmental temperature or operating frequency has a marked effect on the $\varepsilon$ values of DEs (Jean-Mistral et al., 2010; Vu-Cong et al., 2013).

A theoretical way to account for these observations on permittivity variation is to postulate that $\varepsilon$ is a function of system variables, instead of being constant. Consider the case of a quasi-linear DE whose permittivity varies with the principal stretches according to $\varepsilon=\varepsilon\left(\lambda_{1}, \lambda_{2}\right)=D / E$, and its free energy density can be written from Eq. (18) as $W\left(\lambda_{1}, \lambda_{2}, D\right)=W_{\mathrm{s}}\left(\lambda_{1}, \lambda_{2}\right)+\frac{D^{2}}{2 \varepsilon\left(\lambda_{1}, \lambda_{2}\right)}$. Following the similar procedure in Section 2.1, one obtains:

$$
\begin{gathered}
\sigma_{1}-\sigma_{3}=\lambda_{1} \frac{\partial W_{\mathrm{s}}\left(\lambda_{1}, \lambda_{2}\right)}{\partial \lambda_{1}}-\left[\varepsilon\left(\lambda_{1}, \lambda_{2}\right)+\frac{\lambda_{1}}{2} \frac{\partial \varepsilon\left(\lambda_{1}, \lambda_{2}\right)}{\partial \lambda_{1}}\right] E^{2}, \\
\sigma_{2}-\sigma_{3}=\lambda_{2} \frac{\partial W_{\mathrm{s}}\left(\lambda_{1}, \lambda_{2}\right)}{\partial \lambda_{2}}-\left[\varepsilon\left(\lambda_{1}, \lambda_{2}\right)+\frac{\lambda_{2}}{2} \frac{\partial \varepsilon\left(\lambda_{1}, \lambda_{2}\right)}{\partial \lambda_{2}}\right] E^{2} .
\end{gathered}
$$

Specifying the permittivity $\varepsilon\left(\lambda_{1}, \lambda_{2}\right)$ and the strain energy function $W_{\mathrm{s}}\left(\lambda_{1}, \lambda_{2}\right)$ leads to the equations of state for quasi-linear DEs.

The polarization of a DE is generally influenced by the deformation and the permittivity, both depending on the stretches. Li B. et al. (2012) developed a theoretical model to predict the conditional polarization to occur, which reveals the inherent physics of stretch-dependent polarization and its effects on DEs' electromechanical behaviors. In a more recent study, Liu et al. (2014) considered the influence of both temperature and deformation on the dielectric constant by constructing a free energy function based on a thermodynamic system, with results showing that tuning temperature and the ratio between principal planar stretches (i.e., $\lambda_{1} / \lambda_{2}$ ) may enhance the stability of DE materials or structures. 


\section{Viscoelasticity of DEs}

\subsection{Relaxation modes of DEs}

A DE-based device is often subjected to time-varying forces and voltages working as an actuator or a generator (Hong, 2011; Foo et al., 2012a; 2012b; Hu and Suo, 2012; Zhang et al., 2014b). Therefore, the working performance of the DE device can be severely influenced by the undergoing dissipative process within the material. The path of each dissipative process to reach its equilibrium is known as relaxation and it has a characteristic time scale. There are three main relaxation modes of DEs, as follows: dielectric relaxation results from the polarization of the polymer chains of the elastomers; viscoelastic relaxation is attributed to the viscoelastic behaviors of the materials; conductive relaxation is caused by the migration of electrons and ions through the elastomers. These dissipative processes have been experimentally characterized, showing that the dielectric relaxation time of typical DEs is of the order of $10^{-6} \mathrm{~s}$ at room temperature, the viscoelastic relaxation may take minutes, and the conductive relaxation is of the order of hours (Seki et al., 1995; Reffaee et al., 2009; Qu et al., 2012).

In the literature, there is a relative paucity of quantification on conductive relaxation of DEs, mainly because the related time scale is far separated from the other two modes. It is reasonable to assume that the conductive relaxation just begins after the equilibrium of the other two modes is reached. Additionally, the dielectric relaxation is orders of magnitude faster than the viscoelastic relaxation, and as such the dynamic evolution of the dielectric relaxation is often ignored. Therefore, the entire dissipation process is approximated as solely the viscoelastic relaxation (Zhao et al., 2011; Foo et al., 2012a). At various working conditions, the viscoelasticity of a DE can remarkably influence its performance (Zhang et al., 2005; Plante and Dubowsky, 2006). For example, in artificial muscles made of DEs, the dissipation energy due to material viscoelasticity was found to severely reduce their efficiency (Kornbluh et al., 2000; Nguyen et al., 2014; Zhang et al., 2014a). Taking advantage of viscoelasticity, a viscoelastic DE has been shown to enable larger actuation strain than a purely elastic $\mathrm{DE}$, with controlled time-dependence of the applied voltage (Zhao et al., 2011). It has also been shown that the electromechanical instability of a $\mathrm{DE}$ is greatly influenced by its viscoelasticity (Park and Nguyen, 2013; Zhou et al., 2014a).

\subsection{Viscoelasticity of DEs: theory}

Theoretically, there is a general approach that accounts for the viscoelastic behaviors of DE materials based on the principles of non-equilibrium thermodynamics (Hong, 2011; Zhao et al., 2011). Considering the DE membrane in Fig. 1a as viscoelastic, the increase of system free energy in the DE should not exceed the total external work from a thermodynamic viewpoint, namely,

$$
\delta F \leq P_{1} \delta l_{1}+P_{2} \delta l_{2}+P_{3} \delta l_{3}+\phi \delta Q
$$

Dividing both sides by the volume of the membrane, i.e., $L_{1} L_{2} L_{3}$, the thermodynamic inequality in Eq. (24) becomes

$$
\delta W \leq \tilde{\sigma}_{1} \delta \lambda_{1}+\tilde{\sigma}_{2} \delta \lambda_{2}+\tilde{\sigma}_{3} \delta \lambda_{3}+\tilde{E} \delta \tilde{D}
$$

Assuming the DE membrane to be incompressible, the free energy density can be expressed as

$$
W=W\left(\lambda_{1}, \lambda_{2}, \lambda_{3}, \tilde{D}, \xi_{1}, \xi_{2}, \ldots\right)
$$

where $\lambda_{1}, \lambda_{2}, \lambda_{3}$, and $\tilde{D}$ are the kinematic quantities through which the external work is done. In contrast, the additional quantities $\left(\xi_{1}, \xi_{2}, \ldots\right)$ are the internal variables that represent any degrees of freedom associated with the underlying dissipative process. Combining Eqs. (25) and (26) leads to

$$
\begin{aligned}
& \left(\frac{\partial W}{\partial \lambda_{1}}-\tilde{\sigma}_{1}\right) \delta \lambda_{1}+\left(\frac{\partial W}{\partial \lambda_{2}}-\tilde{\sigma}_{2}\right) \delta \lambda_{2}+\left(\frac{\partial W}{\partial \lambda_{3}}-\tilde{\sigma}_{3}\right) \delta \lambda_{3} \\
& +\left(\frac{\partial W}{\partial \tilde{D}}-\tilde{E}\right) \delta \tilde{D}+\sum_{i} \frac{\partial W}{\partial \xi_{i}} \delta \xi_{i} \leq 0 .
\end{aligned}
$$

This thermodynamic inequality holds for arbitrary small changes of the variable set $\left(\lambda_{1}, \lambda_{2}, \lambda_{3}, \tilde{D}, \xi_{1}\right.$, $\left.\xi_{2}, \ldots\right)$. If the DE system is in mechanical and electrostatic equilibrium,

$$
\tilde{\sigma}_{1}=\frac{\partial W\left(\lambda_{1}, \lambda_{2}, \lambda_{3}, D, \xi_{1}, \xi_{2}, \ldots\right)}{\partial \lambda_{1}}
$$




$$
\begin{aligned}
& \tilde{\sigma}_{2}=\frac{\partial W\left(\lambda_{1}, \lambda_{2}, \lambda_{3}, D, \xi_{1}, \xi_{2}, \ldots\right)}{\partial \lambda_{2}}, \\
& \tilde{\sigma}_{3}=\frac{\partial W\left(\lambda_{1}, \lambda_{2}, \lambda_{3}, D, \xi_{1}, \xi_{2}, \ldots\right)}{\partial \lambda_{3}}, \\
& \tilde{E}=\frac{\partial W\left(\lambda_{1}, \lambda_{2}, \lambda_{3}, D, \xi_{1}, \xi_{2}, \ldots\right)}{\partial \tilde{D}},
\end{aligned}
$$

and the inequality reduces to

$$
\sum_{i} \frac{\partial W\left(\lambda_{1}, \lambda_{2}, \lambda_{3}, D, \xi_{i}, \xi_{i}, \ldots\right)}{\partial \xi_{i}} \delta \xi_{i} \leq 0
$$

The inequality in Eq. (32) may be satisfied by selecting a proper relation between $\left(\delta \xi_{1}, \delta \xi_{2}, \ldots\right)$ and $\left(\frac{\partial W}{\delta \xi_{1}}, \frac{\partial W}{\delta \xi_{2}}, \ldots\right)$. For example, one may choose a kinetic model of the type:

$$
\frac{\mathrm{d} \xi_{i}}{\mathrm{~d} t}=-\sum_{j} M_{i j} \frac{\partial W\left(\lambda_{1}, \lambda_{2}, \lambda_{3}, D, \xi_{1}, \xi_{2}, \ldots\right)}{\partial \xi_{j}},
$$

where $M_{i j}$ is a positive-definite matrix which generally depends on the independent variables $\left(\lambda_{1}, \lambda_{2}, \lambda_{3}\right.$, $\left.\tilde{D}, \xi_{1}, \xi_{2}, \ldots\right)$. The elastomer dissipates energy through the changes of the internal variables $\left(\xi_{1}\right.$, $\left.\xi_{2}, \ldots\right)$, with the dissipation rate

$$
-\sum_{i} \frac{\partial W}{\partial \xi_{i}} \frac{\mathrm{d} \xi_{i}}{\mathrm{~d} t}=\sum_{i, j} M_{i j} \frac{\partial W}{\partial \xi_{i}} \frac{\partial W}{\partial \xi_{j}} .
$$

Once a set of internal variables $\left(\xi_{1}, \xi_{2}, \ldots\right)$ is identified, the energy density function $W$ and the matrix $M_{i j}$ determine the dissipative behaviors of the DE.

This approach based on non-equilibrium thermodynamics provides a concise and convenient way to construct the constitutive relations of viscoelastic DEs. There exist a number of other options in choosing the kinetic models that satisfy the thermodynamic inequality in Eq. (32). For example, a rheological model has been adopted to represent a viscoelastic DE as an array of springs and dashpots (Fig. 2) (Zhao et al., 2011; Li T.F. et al., 2012a; Sheng et al., 2013; Wang H. et al., 2013). The two springs in Fig. 2 represent the elasticity of two types of polymer chains in the DE: one being elastic that deforms reversibly, and the other relaxing with time. The viscous deformation is captured by the dashpot. To better understand this non-equilibrium process, one can imagine an intermediate state between the reference state and the current one, which can be achieved by isolating and elastically relaxing spring $\beta$ while maintaining the state of the dashpot $\eta$, as illustrated in Fig. 2 (Hong, 2011). In the current state, both of the two springs are stretched and contribute to the load-carrying capacity. The total strain is the sum of the elastic strain in spring $\beta$ and the inelastic strain in dashpot $\eta$, and the total stress that corresponds to the elastic part becomes the driving force of the viscous deformation in the dashpot. In the virtual intermediate state, the mechanical load is sustained by spring $\alpha$ only, and the key difference between the intermediate and current states lies in the elastic strain in spring $\beta$. This simple rheological representation actually captures the essence of DE viscoelasticity in many studies (Zhao et al., 2011; Wang Y.Q. et al., 2013; Zhang et al., 2014a).

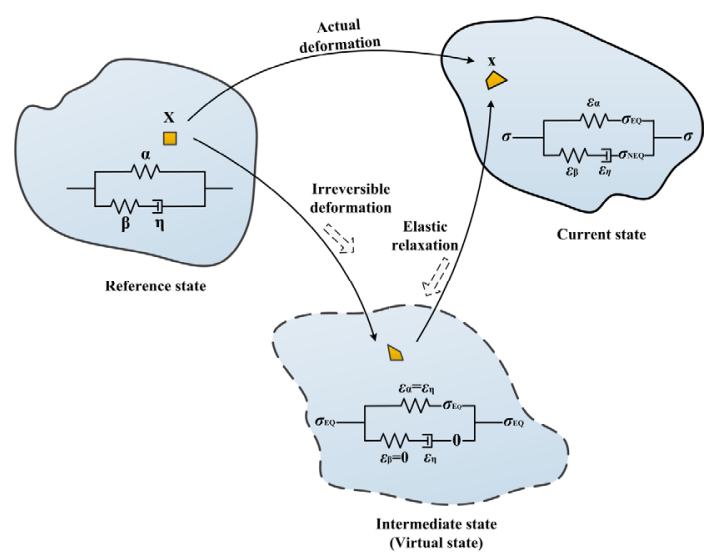

Fig. 2 A rheological model of DEs

The viscoelasticity model consisting of two components in parallel: one being a spring $\alpha$, and the other including another spring $\beta$ and a dashpot $\eta$. An arbitrary deformation can be decomposed into two parts by postulating an intermediate state of deformation, which is achieved by elastic relaxation of spring $\beta$ from the current state (Hong, 2011; Zhao et al., 2011; Wang Y.Q. et al., 2013)

Using the rheological model in Fig. 2 and the neo-Hookean free energy density description, Zhao et al. (2011) calculated the critical condition for electromechanical instability to occur, suggesting that one can achieve a larger deformation of actuation in a viscoelastic DE than a purely elastic one if the time-dependence of the applied voltage is carefully 
controlled. Similarly, Zhang et al. (2014b) presented a dissipative model of a viscoelastic DE and investigated the influence of dissipation on its dynamic performance, in comparison with the effects of current leakage. Their results suggest that the material viscoelasticity effectively reduces the natural frequency and increases the mean stretch of the DE, while the current leakage influences the DE behaviors in an opposite manner. Wang Y.Q. et al. (2013) developed a visco-hyper-elastic constitutive relationship based on the rheological model in Fig. 2, with quantitative predictions on the hysteresis and energy dissipation in the DE film.

\subsection{Computational methods of DE viscoelasticity}

Most analytical studies on DE viscoelasticity have been limited to axisymmetric configurations, such as circles, cylinders, spheres, etc. (Wang H. et al., 2013), and numerical methods are often required in dealing with more complicated or irregular shapes, such as those DE structures in practical applications (Hong, 2011; Wang et al., 2012). A comprehensive nonlinear theory based on field description has been developed to describe the deformation and electric fields of DEs under externally applied forces as well as electric voltage (Reese and Govindjee, 1998; Goulbourne et al., 2007; Suo et al., 2008), which serves as a theoretical foundation for finite element implementation.

Following a continuum-level approach (Suo, 2010; Bueschel et al., 2011; Park et al., 2012), let us focus on an arbitrary material particle at position $\boldsymbol{X}$ in the reference state. At time $t$, it moves to a new place $\boldsymbol{x}$ which is a function of $\boldsymbol{X}$ and $t$, i.e., $\boldsymbol{x}=\boldsymbol{x}(\boldsymbol{X}, t)$, referring to an arbitrary point in Fig. 2. The deformation gradient is defined through

$$
F_{i j}=\frac{\partial x_{i}(\boldsymbol{X}, t)}{\partial X_{j}}
$$

At time $t$, let $\phi(X, t)$ be the electric potential of material particle $\boldsymbol{X}$ at time $t$. The nominal electric field is therefore

$$
\tilde{E}_{i}=\frac{\partial \phi(X, t)}{\partial X_{i}}
$$

The nominal stress $\tilde{\sigma}_{i j}$ has to satisfy the weak form of the momentum balance equation, namely,

$$
\int \tilde{\sigma}_{i j} \frac{\partial \chi_{i}}{\partial X_{j}} \mathrm{~d} V=\int\left(B_{i}-\rho \frac{\partial^{2} x_{i}}{\partial t^{2}}\right) \chi_{i} \mathrm{~d} V+\int T_{i} \chi_{i} \mathrm{~d} A
$$

where $\chi_{i}(\boldsymbol{X})$ is an arbitrary vector test function, $B_{i}$ is the body force per unit reference volume $V, \rho$ is the mass density of the material, and $T_{i}$ is the force per unit area that is applied to the material surface in the reference configuration. In Eq. (37), the repeated indices (i.e., $i$ and $j$ ) imply summation from 1 to 3 .

Similarly, the nominal electric displacement $\tilde{D}_{i}$ should satisfy the following weak form of governing equation:

$$
-\int \tilde{D}_{i} \frac{\partial \eta}{\partial X_{i}} \mathrm{~d} V=\int q \eta \mathrm{d} V+\int \omega \eta \mathrm{d} A
$$

where $\eta(\boldsymbol{X})$ is an arbitrary scalar test function, $q$ is the volumetric charge density, and $\omega$ is the surface charge density. Both $q$ and $\omega$ are measured with respect to the reference configuration. All the integrals in Eqs. (37) and (38) are carried out over the entire volume or surface under investigation.

To supplement Eqs. (35)-(38) for a complete set of formulae as a boundary value problem (Wang J. et al., 2014), one needs to specify the material laws that describe the underlying electromechanical coupling within DEs. This has been achieved by many researchers using the variational method (Suo, 2010; Khan et al., 2013; Lu et al., 2013; 2014; Wang J. et al., 2014), and finite element methods have been developed and used to explore the electromechanical behaviors of various DE transducers (Qu and Suo, 2012). Park and coworkers (Park et al., 2012; Park and Nguyen, 2013) developed a viscoelastic finite element formulation with focus on the finite and dynamic deformation of DE structures. Their results demonstrated significant effects of viscoelasticity on the dielectric behaviors of elastomers that may undergo electromechanical instabilities caused by homogeneous as well as inhomogeneous deformations. Khan et al. (2013) formulated a numerical framework based on the variational method and studied the nonlinear viscous behavior of DEs. The generalized Maxwell model was adopted in performing a series of simulations on different types of electrically driven 
actuators under various electromechanical loadings, with the prediction that electromechanical instability can be avoided if the DE actuators operate at a high voltage rate under a dead load (Khan et al., 2013).

The electromechanical response of a DE is strongly coupled to its viscoelastic behaviors. The existing numerical methods have provided powerful and reliable tools to study DE behaviors in many aspects, such as nonlinear viscoelasticity, timedependent effects, large strains, and electromechanical coupling effects (Bueschel et al., 2013). However, there still exist unsolved problems in investigating the rheological behavior of DEs, which call for more sophisticated treatments of the computational approach in the future.

\section{Electromechanical instability vs. large deformation}

A desirable DE actuator is expected to produce large actuation strain under relatively low voltage ( $\mathrm{Li}$ T.F. et al., 2014b). A deeper understanding of various failure modes should allow for the design of DE-based actuators capable of operating in a wider and safer regime with prolonged lifetime. In early studies of DE actuation, Pelrine et al. (1998) found that an actuation strain of about $30 \%$ can be achieved in silicone elastomers coated with compliant electrodes. Later research showed that pre-straining DE films can enhance the actuation strain up to $\sim 100 \%$ (Pelrine et al., 2000). Since that time, various means have been tried to improve the actuation performance of DE devices. For example, mixing the elastomers with penetrating networks, swelling the elastomers with a solvent, and pre-stretching the elastomers were all found to effectively enhance DEs' actuation strain (Ha et al., 2006; Suo and Zhu, 2009; Goulbourne, 2011). However, some outstanding theoretical questions still exist: what are the underlying mechanisms causing such enhancement? Is there an upper limit for the actuation strain? If such a limit exists, how to optimize the system parameters to achieve the limit?

\subsection{Electromechanical instability of DEs}

In pursuing the limit of large deformation by an externally applied voltage, DEs may suffer several failure modes that ultimately limit the actuation per- formance with respect to homogeneous as well as inhomogeneous deformations. Two failure modes occur most frequently: electrical breakdown and electromechanical instability. The latter is also known as pull-in instability as a result of the mutual promotion between the electric voltage and voltage-induced deformation in compliant DEs. Referring to the membrane configuration in Fig. 1, as a voltage is applied to the DE membrane, the electrostatic attraction between the electrodes thins down the elastomer, which in turn causes a higher electric field. This positive feedback can catastrophically fail the elastomer in turnovers of deformation increase and electric field amplification under some circumstances, also known as premature electrical breakdown (Zhao et al., 2007; Kolloscheet al., 2012).

Consider the DE transducer and accompanying external loadings, including the force and voltage, as a thermodynamic system. The preceding Eqs. (5)-(8) constitute the states of the material, which can be reformed in the incremental form as

$\left[\begin{array}{c}\frac{\mathrm{d} \tilde{\sigma}_{1}}{\mathrm{~d} t} \\ \frac{\mathrm{d} \tilde{\sigma}_{2}}{\mathrm{~d} t} \\ \frac{\mathrm{d} \tilde{\sigma}_{3}}{\mathrm{~d} t} \\ \frac{\mathrm{d} \tilde{E}}{\mathrm{~d} t}\end{array}\right]=\left[\begin{array}{cccc}\frac{\partial^{2} W}{\partial \lambda_{1}^{2}} & \frac{\partial^{2} W}{\partial \lambda_{1} \partial \lambda_{2}} & \frac{\partial^{2} W}{\partial \lambda_{1} \partial \lambda_{3}} & \frac{\partial^{2} W}{\partial \lambda_{1} \partial \tilde{D}} \\ \frac{\partial^{2} W}{\partial \lambda_{2} \partial \lambda_{1}} & \frac{\partial^{2} W}{\partial \lambda_{2}^{2}} & \frac{\partial^{2} W}{\partial \lambda_{2} \partial \lambda_{3}} & \frac{\partial^{2} W}{\partial \lambda_{2} \partial \tilde{D}} \\ \frac{\partial^{2} W}{\partial \lambda_{3} \partial \lambda_{1}} & \frac{\partial^{2} W}{\partial \lambda_{3} \partial \lambda_{2}} & \frac{\partial^{2} W}{\partial \lambda_{3}^{2}} & \frac{\partial^{2} W}{\partial \lambda_{3} \partial \tilde{D}} \\ \frac{\partial^{2} W}{\partial \tilde{D} \partial \lambda_{1}} & \frac{\partial^{2} W}{\partial \tilde{D} \partial \lambda_{2}} & \frac{\partial^{2} W}{\partial \tilde{D} \partial \lambda_{3}} & \frac{\partial^{2} W}{\partial \tilde{D}^{2}}\end{array}\right]\left[\begin{array}{c}\frac{\mathrm{d} \lambda_{1}}{\mathrm{~d} t} \\ \frac{\mathrm{d} \lambda_{2}}{\mathrm{~d} t} \\ \frac{\mathrm{d} \lambda_{3}}{\mathrm{~d} t} \\ \frac{\mathrm{d} \tilde{D}}{\mathrm{~d} t}\end{array}\right]$,

through which the Hessian matrix of free-energy function is identified as

$$
\boldsymbol{H}=\left[\begin{array}{cccc}
\frac{\partial^{2} W}{\partial \lambda_{1}^{2}} & \frac{\partial^{2} W}{\partial \lambda_{1} \partial \lambda_{2}} & \frac{\partial^{2} W}{\partial \lambda_{1} \partial \lambda_{3}} & \frac{\partial^{2} W}{\partial \lambda_{1} \partial \tilde{D}} \\
\frac{\partial^{2} W}{\partial \lambda_{2} \partial \lambda_{1}} & \frac{\partial^{2} W}{\partial \lambda_{2}^{2}} & \frac{\partial^{2} W}{\partial \lambda_{2} \partial \lambda_{3}} & \frac{\partial^{2} W}{\partial \lambda_{2} \partial \tilde{D}} \\
\frac{\partial^{2} W}{\partial \lambda_{3} \partial \lambda_{1}} & \frac{\partial^{2} W}{\partial \lambda_{3} \partial \lambda_{2}} & \frac{\partial^{2} W}{\partial \lambda_{3}^{2}} & \frac{\partial^{2} W}{\partial \lambda_{3} \partial \tilde{D}} \\
\frac{\partial^{2} W}{\partial \tilde{D} \partial \lambda_{1}} & \frac{\partial^{2} W}{\partial \tilde{D} \partial \lambda_{2}} & \frac{\partial^{2} W}{\partial \tilde{D} \partial \lambda_{3}} & \frac{\partial^{2} W}{\partial \tilde{D}^{2}}
\end{array}\right] .
$$


The equilibrium of the thermodynamic system requires $\boldsymbol{H}$ to be positive definite (Zhang et al., 2012; Zhao and Wang, 2014), and the DE transducer becomes unstable whenever the determinant of $\boldsymbol{H}$ vanishes. It should be noticed that $\operatorname{det}(\boldsymbol{H}) \neq 0$ does not distinguish stable and metastable cases, because the Hessian matrix is positive-definite in either of them. Experiments showed that a pre-stretched DE membrane deforms into a complex pattern under a critical level of voltage, where two states, one being flat and the other being wrinkled, coexist (Plante and Dubowsky, 2006; Li T.F. et al., 2014a; Zou et al., 2014). To determine the stable state, one needs to globally search for the system minimum in terms of its free energy (Zhao et al., 2007; Huang and Suo, 2012).

\subsection{Three types of DE transducers}

Consider an ideal and incompressible DE membrane in its reference state (Fig. 1a). Now assume that the membrane is of unit area and has thickness $H$. Upon an applied voltage, the membrane is stretched by $\lambda$ in two perpendicular in-plane directions and is shrunk by $\lambda^{2}$ in the out-of-plane direction, as shown in Fig. 3a. The thickness of the membrane reduces to $H \lambda^{-2}$, and the electric field in the membrane is simply $E=\lambda^{2} \phi / H$. A typical DE exhibits a stress-stretch curve $\sigma(\lambda)$ with the following features: the DE is relatively compliant at small stretches, and it stiffens at modest stretches before approaching the stretch limit $\lambda_{\text {lim }}$.

An important quantity in $\mathrm{DE}$ actuation is the Maxwell stress $\varepsilon E^{2}$. In the absence of externally applied mechanical load, the equation of state recovers $\sigma(\lambda)=\varepsilon E^{2}$. The voltage is therefore related to the stretch as

$$
\phi=H \lambda^{-2} \sqrt{\sigma(\lambda) / \varepsilon}
$$

In contrast to the stress-stretch curve $\sigma(\lambda)$ that is usually monotonic, the voltage-stretch curve $\phi(\lambda)$ is not (Zhao et al., 2007). The shape of $\phi(\lambda)$ can be readily interpreted from Eq. (41): at small stretches $(\lambda \sim 1)$, the increase from the term $\sqrt{\sigma(\lambda)}$ suppresses the decrease in $\lambda^{-2}$, and the overall voltage increases with the stretch; at intermediate stretches, $\lambda^{-2}$ becomes the dominating term during the thinning of the membrane, and thus the voltage curve falls down as the stretch increases, which is exhibited as pull-in instability because of the positive feedback between a thinning $\mathrm{DE}$ and an increasing electric field; as the DE membrane approaches the stretch limit $\lambda_{\text {lim }}$, the curve of $\sigma(\lambda)$ rises, and so does the voltage curve $\phi(\lambda)$.

The critical voltage $\phi_{B}$ for the electric breakdown is also a function of stretch. Experiments showed that $\phi_{B}$ is a monotonically decreasing function of the stretch $\lambda$ (Kofod et al., 2003; Koh et al., 2011; Suo, 2012). Depending on where the two curves of $\phi(\lambda)$ and $\phi_{B}$ intersect, DE transducers can be classified into three types (Zhao and Suo, 2007; Suo, 2010; Li et al., 2011), as illustrated in Figs. 3a and 3b. A type I transducer suffers electrical breakdown as the $\phi(\lambda)$ curve intersects the $\phi_{B}$ curve before the occurrence of electromechanical instability, which only produces a small deformation of actuation. A type II transducer passes the peak of the $\phi(\lambda)$ curve before the electric breakdown, and this type of DE transducer is recorded to fail at the peak of $\phi(\lambda)$, which can be much lower than the breakdown voltage $\phi_{B}$. A type III transducer reaches a stable state before the electrical breakdown, thereby avoiding electromechanical instability and attaining a large voltageinduced deformation.

\subsection{Influence of pre-stretch on DE performance}

Experiments have shown that electromechanical instability can be eliminated by pre-stretch, as exemplified by Mao et al. (2014) showing that prestretch along the axis of a tubular DE balloon can suppress the snap-through instability during inflation and regulate the reaction force along the axial direction. More optimized electromechanical performance of DE actuators can be achieved by controlling the form and distribution of pre-stress (Zhao and Suo, 2007; Zhao et al., 2007). These phenomena can be easily understood by using the DE membrane model depicted in Fig. 1: a pre-applied external force $P_{\text {pre }}$ gives rise to a mechanical stress $\sigma_{\mathrm{pre}}=P_{\mathrm{pre}} \lambda / H$. Combining this pre-applied mechanical stress and the Maxwell stress, both causing the DE membrane to deform, we have

$$
\sigma_{\text {pre }}+\varepsilon E^{2}=\sigma(\lambda)
$$

The voltage can be expressed as 


$$
\phi=\frac{H \lambda^{-2}}{\sqrt{\varepsilon}} \sqrt{\sigma(\lambda)-P_{\mathrm{pre}} \lambda / H} .
$$

As illustrated in Fig. 3c, $P_{\text {pre }}$ has two effects on the $\phi(\lambda)$ curve: it first induces an initial strain in the elastomer and moves the original point closer to $\lambda_{\text {lim }} ; P_{\text {pre }}$ also reduces the required voltage $\phi$ for actuation, referring to Eq. (43). Prior to the voltage application, a prestretched elastomer is thinner, and therefore the voltage $\phi$ required to induce the same electric field in a pre-stretched elastomer is lower than that of an unstrained elastomer (Lu et al., 2012). As such, prestretch can significantly enhance the actuation performance of DEs and alleviate the occurrence of electromechanical instability.

The level of pre-stretch is not the sole factor that may eliminate the electromechanical instability and enhance DEs' actuation strain; the voltage-induced deformation is also strongly affected by how the mechanical loads are preset (Li T.F. et al., 2012b; Lu et al., 2012; Godaba et al., 2014; Zhou et al., 2014b). In fact, the electromechanical responses of DEs are different under uniaxial, biaxial, and shear forces (Lochmatter et al., 2007; Lu et al., 2012; Huang et al., 2013; Wang Y. et al., 2013): large voltage-induced deformation has been demonstrated for a membrane under equal-biaxial forces, while much smaller voltage-induced deformation can be achieved for a membrane under a uniaxial force; pure shear was shown to cause decreased tensile force as the voltage increases (Qu et al., 2012; Wang Y. et al., 2013; 2014a). These results showed that the electromechanical performance of a DE can be tuned by manipulating how the mechanical loading is applied before electric voltage (Zhou et al. 2013), and theoretical analyses provide insight and guidance in designing such DE structures and devices. Huang et al. (2012) recently demonstrated that voltage-induced expansion could reach $488 \%$ of areal strain when a DE membrane is subjected to biaxial dead loads. Keplinger et al. (2012) and Li T.F. et al. (2013) were able to achieve a giant voltage-triggered expansion of area by $1692 \%$ in a DE membrane by harnessing the electromechanical instability with air pressure control.

It should be pointed out that the preceding results in Eqs. (39)-(43) on electromechanical instability are based on the simple configuration of a planar DE membrane sandwiched between two compliant electrodes, where the deformation and electric fields are homogeneous. In the presence of 3D DEs (such as many of the applications in the following section), inhomogeneous fields occur and may cause multiple failure modes at localized points. For example, failure modes including electrical breakdown, loss of tension and rupture by stretch have been illustrated for a DE membrane deformed into an out-of-plane axisymmetric shape (He et al., 2009) and an inflated DE actuator with tubular configuration (Zhang et al., 2015). Localized electromechanical instabilities were demonstrated by observing voltage-induced wrinkling in a DE strip (Park et al., 2012) and an inflated DE balloon (Mao et al., 2015). A spherical DE balloon under internal pressure and electric voltage has been analyzed, with results showing that inhomogeneous viscoelastic deformation may cause timedependent failure modes (Wang H. et al., 2013), and bifurcation to a non-spherical shape may occur under certain electromechanical loading conditions (Liang and Cai, 2015).
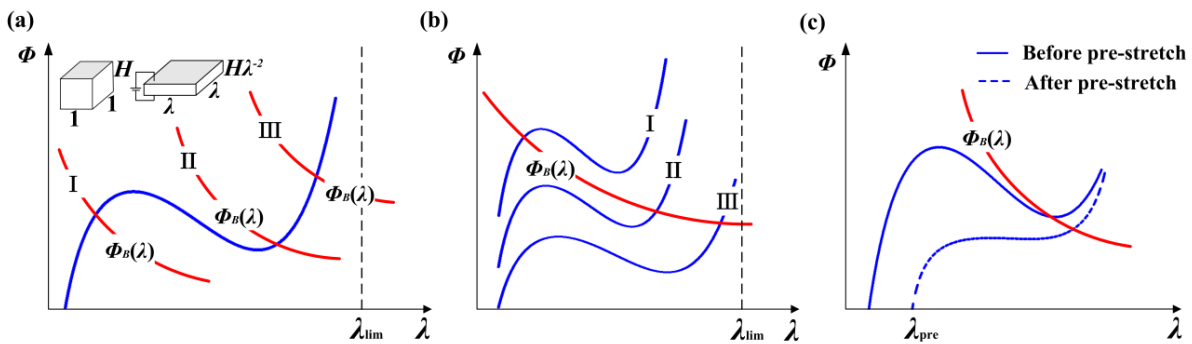

Fig. 3 Three types of DE transducers and the influence of pre-stretch on electromechanical instability (a) and (b) Three types of dielectrics, depending on where the two curves $\phi(\lambda)$ and $\phi_{B}(\lambda)$ intersect; (c) Electromechanical response of a DE membrane with or without pre-stretch 


\section{Applications of DE actuation}

The exploration to replace existing electromagnetic actuators, pneumatics, piezo-actuators as well as natural muscles with DE actuators has been under continuous progress since the early 2000s (Pelrine et al., 2000), and many prototype applications already exist with various configurations over a wide range of dimensions. Common configurations of DE-based actuators are routinely classified as film, tube, rolled, frame-supported and laminated. Robots made of these DE devices have been demonstrated to have the capability of sophisticated locomotion, and theoretical and numerical studies played a fundamental role in realizing these applications. Table 2 summarizes the key attributes, such as actuation strain and stress, strain rate, work density, response time, etc., which quantify the performance and effectiveness of typical $\mathrm{DE}$ actuators in comparison to natural muscles. Although work is still far from achieving the delicate motions of natural muscles in artificial DE devices, the exploration of various practical applications is progressing in many ways. In the following, we will introduce some representative examples of DE devices that are already in use.
The actuation of DEs is inevitably accompanied by the generation of forces and change in shape. Area expansion of DE films has been used in actuation. An interesting example is the following: to mimic fish-like movement in air (Jordi et al., 2010), pre-stretched actuators were mounted on both sides of an airship hull. When one actuator expands due to electric activation, the other one contracts at the same time, and a net bending motion is generated to drive the flying body forward. As another example, Fig. 4a shows the working principle of a fluid-filled elastomeric lens with an annular DE actuator as an artificial muscle. A voltage between 1 to $3 \mathrm{kV}$ was applied to the annular actuator to deform the lens and it successfully changed the focal length, with multiple advantages such as silent operation, compact size, and low weight (Jung et al., 2011). DE-activated tunable optics like transmission grating and structural color modulators also make use of the voltage-induced area expansion of DEs as the main activation mechanism (Wang Y. et al., 2014b).

DE actuators in diaphragm configuration usually induce out-of-plane deformation by applying electric voltage to a properly constrained $\mathrm{DE}$ membrane, as indicated in Fig. 4b (Vertechy et al., 2012; Ahmed

Table 2 Actuation performance: natural muscles vs. DEs

\begin{tabular}{|c|c|c|}
\hline Property & Natural muscles & DEs \\
\hline Energy density & $\begin{array}{l}150 \mathrm{~J} / \mathrm{kg} \text { (peak to } 300 \mathrm{~J} / \mathrm{kg} \text { ) } \\
\text { (Boy et al., 2013) }\end{array}$ & $3400 \mathrm{~J} / \mathrm{kg}$ (Brochu and Pei, 2010) \\
\hline $\begin{array}{l}\text { Frequencies and response } \\
\text { time }\end{array}$ & $10 \mathrm{~Hz}, 10 \mathrm{~ms}$ (Meijer et al., 2001) & $10 \mathrm{kHz}, 0.1 \mathrm{~ms}$ (Sheng et al., 2013) \\
\hline Maximum strain & 100\% linear (Brochu and Pei, 2010) & $1692 \%$ areal (Li T.F. et al., 2013) \\
\hline Efficiency & - & $60 \%-90 \%$ (Brochu and Pei, 2010) \\
\hline Stroke & $20 \%-40 \%$ linear (peak to $100 \%$ ) & $\begin{array}{l}\text { 10\%-100\% linear (peak to } 300 \%) \\
\text { (O'Halloran et al., 2008) }\end{array}$ \\
\hline Actuation mechanism & Molecular motors & Maxwell force \\
\hline Power source & ATP & $\begin{array}{l}\text { Electricvoltage }(1-10 \mathrm{kV}) \\
\quad \text { (Shankar } \text { et al., 2007) }\end{array}$ \\
\hline Environment & Wet and nutrition & Insulation \\
\hline Structure & Bundled muscle cells, self-assembly & $\begin{array}{l}\text { Sandwiched membrane, grouped tensegrity } \\
\text { and electrodes arrays (Bauer et al., 2014) }\end{array}$ \\
\hline Size & $10 \mu \mathrm{m}-1 \mathrm{~m}$ (Magid and Law, 1985) & $100 \mu \mathrm{m}-0.1 \mathrm{~m}$ (Brochu and Pei, 2010) \\
\hline Fabrication & Tissue engineering & Lithography, machining \\
\hline Typical material & $\begin{array}{l}\text { Mammal muscle cells, molecules con- } \\
\text { nected by extracellular matrices }\end{array}$ & $\begin{array}{l}\text { 3M-VHB, PDMS, Silicon } \\
\text { (Li T.F. et al., 2012a; Wang J. et al., 2014) }\end{array}$ \\
\hline Modulus & 20-2000 kPa (Morrow et al., 2010) & $20-2000 \mathrm{kPa}$ (Sheng et al., 2012) \\
\hline Viscoelasticity & 0.1-100 s (van Loocke et al., 2008; 2009) & 0.1-100 s (Zhang et al., 2014a) \\
\hline Fatigues and life time & $10^{7}$ cycles (van Loocke et al., 2009) & $10^{7}$ cycles (Rosset et al., 2009) \\
\hline Density & 1.06 kg/L (Kornbluh et al., 2002) & $0.94 \mathrm{~kg} / \mathrm{L}$ (Shankar et al., 2007) \\
\hline
\end{tabular}




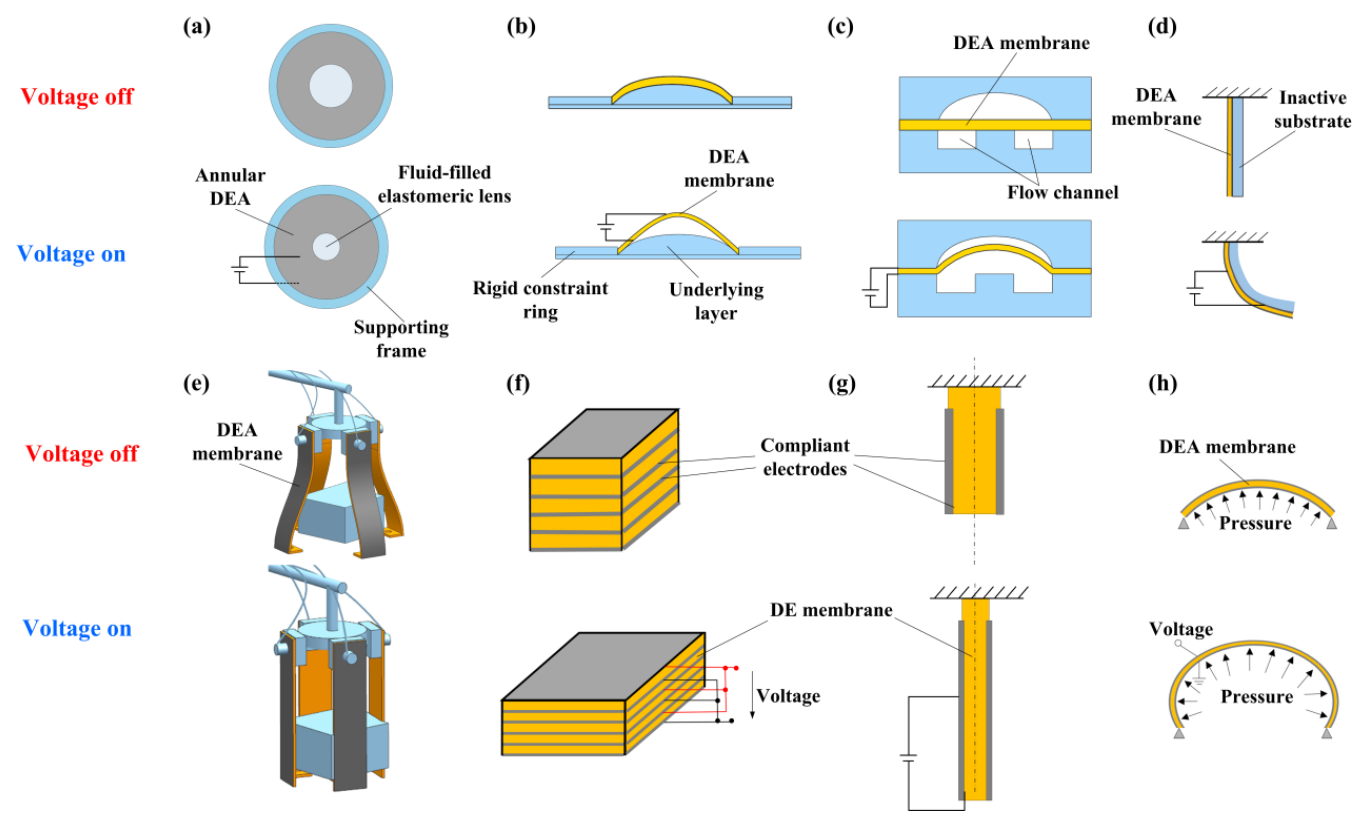

Fig. 4 Various DE actuators and their applications

(a) An eye-camera system made of DE; (b) A bulging DE actuator; (c) A microfluidic valve that is dielectrically actuated; (d) A unimorph DE actuator; (e) A multi-finger DE gripper; (f) A stacking DE actuator; (g) A tubular DE actuator; (h) A swelling DE balloon

et al., 2014). This type of DE actuator usually consists of an initially curved membrane made of a circular membrane with its boundary constrained to a rigid frame. With an external voltage, the DE membrane bulges as a result of expanding area, with applications as pumps, loud-speakers, and electric generators. Inspired by octopus suckers, Follador et al. (2014) recently designed a novel suction cup using DEs, and this actuator was able to produce up to $6 \mathrm{kPa}$ of pressure in water with a response time of less than $300 \mathrm{~ms}$. DE actuators also have been widely used in microfluidic flow control and large-scale integrated microfluidic chips. These chips consist of arrays of independently controlled pneumatic actuators that can produce pumping or valving action individually (Duncan et al., 2013; Maffli et al., 2013). DE-made actuators can significantly reduce the size of the off-chip components, being a promising candidate to replace the traditional pneumatic powered actuators (Fig. 4c).

Different boundary constraints lead to various deformation modes of $\mathrm{DE}$ devices. A bending motion can be realized by restricting the in-plane deformation of film- or membrane-like structures. A unimorph actuator was made by an electroactive DE layer attached to an inactive substrate (Ahmed et al., 2014), and switching the polarity of active electrodes can produce bending deformation in opposite directions (Fig. 4d). With further sophistication of the substrate and coated electrodes, a bimorph actuator was demonstrated to lift or drop a graphite/epoxy rod (Bar-Cohen, 2004). By carefully devising the elasticity and energy in bending frames and a linear actuator, Kofod et al. (2007) fabricated a DE-gripper which serves as a biomimetic representation of a robotic arm in response to an external voltage (Fig. 4e). A spring-roll bending actuator made of DE was designed by Li J.R. et al. (2014) which can serve as a robotic leg.

Most of the above-mentioned examples make use of the in-plane or out-of-plane deformation of DEs in achieving muscle-like contraction for thin structures such as films or membranes. The actuators in stacking or multi-layer configuration were shown to provide larger forces in parallel and nearly linear contractile response in the thickness direction, as indicated in Fig. 4f (Brochu and Pei, 2010; Haus et al., 2013; Tutcuoglu and Majidi, 2014). Carpi et al. (2005) designed a contractile DE actuator of helical stack configuration, which was capable of 5\% strain under an electric field of $14 \mathrm{~V}$ per $\mu \mathrm{m}$. Alternatively, Kovacs et al. (2009) produced an actuator by pile-up of 
folded actuators, which were $85 \mathrm{~mm}$ high and $25 \mathrm{~mm}$ wide containing layers $0.5-0.8 \mathrm{~mm}$ thick. The contractile motion of the stacked configuration makes it practically suitable for light, compliant, and flexible systems for noise-free actuation. It should be mentioned that there is also a major drawback to this multi-layer configuration, because polymeric stacking actuators with flexible electrodes may have nonuniform distribution of strain along their length. Kaal and Herold (2013) proposed a numerical model to look for an optimal electrode design for stacking DE actuators, and their results showed that a stacking DE actuator with perforated and rigid electrodes exhibits an improved performance. Niu et al. (2013) fabricated buckling actuators that were free of pre-strain to alleviate local stress concentration and other detrimental effects in the elastomers, and their multi-layer structures could shorten by $11 \%$ upon electric activation.

DE actuators of tubular form have also been widely used. A DE tube was made by fixing one end and coating the inner and outer surfaces with compliant electrodes, as shown in Fig. 4g (Carpi and de Rossi, 2004; Zhu et al., 2010). When an external voltage is applied, the $\mathrm{DE}$ tube exhibits a reduction in its thickness accompanied by an elongation in the axial direction. This type of actuation in both axial and radial directions makes it possible for peristaltic pumps (Wu et al., 2012). If the size of such tube-like DE actuators is sufficiently small, it can function as a fiber actuator that may be used as a building block in hierarchical structures of artificial muscles. By incorporating these fiber actuators in conventional textiles, people may also produce electroactive structures for particular purposes (Arora et al., 2007).

There are more configurations of DE actuators such as balloons (Fig. 4h) and rolls, which work at different geometries and boundary conditions but share the same principle of voltage-triggered deformation. The combination of various configurations can definitely provide DE structures or devices with multi-degrees of freedom upon actuation, where the only limitation is the designer's imagination.

\section{Concluding remarks and outlook}

This survey has focused on the approaches and issues of improving DE performance and reliability from a mechanics point of view. These mechanical studies of DE-based materials, structures, and devices provide an excellent opportunity for researchers with backgrounds in mechanics and materials science to work together. A continuous effort from the materials community is to achieve better DEs with increasing dielectric constant. One preferred strategy is to places elected particles that carry high permittivity into the elastomers (Galantini et al., 2013; Liu H. et al., 2013; Tian et al., 2014). Different from the traditional DEs, the effective electromechanical coupling in these particle/matrix composites becomes highly nonlinear, making it a challenging task to properly model such materials from theoretical point of view (Li B. et al., 2013). Some analytical efforts have been devoted to this problem, but most of them were limited to small strains (Tian et al., 2012). Although numerous DE structures and devices have been successfully modeled and analyzed, DE actuators with self-healing capability (Liu Y. et al., 2013), increasing sophistication in stacking or even hierarchical configurations (Haus et al., 2013) require more theoretical effort to address the underlying challenges that impede the practical application of these smart materials in artificial muscles.

The continuous development of theoretical and numerical studies provides a deeper understanding of the underlying electromechanical principles of DEs, which is an essential prerequisite for the design of more powerful and efficient products with long-term stability and durability. It is also a challenge to develop a hierarchical organization of DE-based sensors and actuators that can respond to external stimuli in a programmable and intelligent way. Modeling such hierarchical compartmentalization generally requires theories of large deformation strongly coupled to other fields such as electronics, as well as microscopic processes such as bonding kinetics (Sarangapani et al., 2011; Ju et al., 2015) and mass transport (Jiang et al., 2015) that ubiquitously occur within materials and at interfaces. The analytical and numerical approach based on continuum mechanics and thermodynamics will continue to play an enormous role in understanding these materials, and in modeling these structures and designing these devices (Chen, 2014), and the bottom-up investigation of the microscopic processes in response to external stimuli has just begun. 
One major disadvantage of DEs that limits their applications is the $\mathrm{kV}$-level voltage of operation. In a recent study, synthesis of silicone elastomers with optimized molecular weight, cross-linker, and silica has produced DEs with high permittivity and large actuation strain, which can repeatedly function at an electric field as low as $10.8 \mathrm{~V}$ per $\mu \mathrm{m}$ (Dünki et al., 2015). Interestingly, atomic force microscopy-based studies have demonstrated that the energy density of individual dormant cells (i.e., Bacillus spores) in response to cyclic humidity variations is at least one order of magnitude higher than any synthetic materials including DEs (Chen et al., 2014). Live cells were shown to volumetrically shrink or swell via cross-membrane ion transport upon applied mechanical pressure (Hui et al., 2014). These results highlight the distinct advantage of involving biologically based materials in DE actuation and energy harvesting, and open a new avenue to build DE-made soft machines with tunable mechanical, chemical as well as electrical properties to generate active force/ motion at the (sub)cellular scale for potential applications in biomaterials and biointerfaces (Qian et al., 2013; Zhang et al., 2013).

\section{References}

Ahmed, S., Ounaies, Z., Frecker, M., 2014. Investigating the performance and properties of dielectric elastomer actuators as a potential means to actuate origami structures. Smart Materials and Structures, 23(9):094003.

Anderson, I.A., Gisby, T.A., McKay, T.G., et al., 2012. Multifunctional dielectric elastomer artificial muscles for soft and smart machines. Journal of Applied Physics, 112(4):041101. http://dx.doi.org/10.1063/1.4740023

Arora, S., Ghosh, T., Muth, J., 2007. Dielectric elastomer based prototype fiber actuators. Sensors and Actuators A: Physical, 136(1):321-328.

http://dx.doi.org/10.1016/j.sna.2006.10.044

Bar-Cohen, Y., 2004. Electroactive polymers (EAP) as actuators for potential future planetary mechanisms. 2004 NASA/DoD Conference on Evolvable Hardware, Proceedings, IEEE, p.309-317. http://dx.doi.org/10.1109/EH.2004.1310845

Bauer, S., Bauer-Gogonea, S., Graz, I., et al., 2014. 25th Anniversary Article: A soft future: from robots and sensor skin to energy harvesters. Advanced Materials, 26(1): 149-162. http://dx.doi.org/10.1002/adma.201303349

Boy, C.C., Vanella, F.A., Lattuca, M.E., et al., 2013. Effect of starvation on growth rate, muscle growth and energy density of puyen, Galaxias maculatus. Journal of Applied
Ichthyology, 29(5):1001-1007.

http://dx.doi.org/10.1111/jai.12147

Boyce, M.C., Arruda, E.M., 2000. Constitutive models of rubber elasticity: a review. Rubber Chemistry and Technology, 73(3):504-523.

http://dx.doi.org/10.5254/1.3547602

Brochu, P., Pei, Q., 2010. Advances in dielectric elastomers for actuators and artificial muscles. Macromolecular Rapid Communications, 31(1):10-36. http://dx.doi.org/10.1002/marc.200900425

Bueschel, A., Klinkel, S., Wagner, W., 2011. A viscoelastic model for dielectric elastomers based on a continuum mechanical formulation and its finite element implementation. Proc. SPIE 7976, Electroactive Polymer Actuators and Devices (EAPAD), No. 79761R. http://dx.doi.org/10.1117/12.880283

Bueschel, A., Klinkel, S., Wagner, W., 2013. Dielectric elastomers-numerical modeling of nonlinear viscoelectroelasticity. International Journal for Numerical Methods in Engineering, 93(8):834-856. http://dx.doi.org/10.1002/nme.4409

Carpi, F., de Rossi, D., 2004. Dielectric elastomer cylindrical actuators: electromechanical modelling and experimental evaluation. Materials Science \& Engineering $C$ Biomimetic and Supramolecular Systems, 24(4): 555-562. http://dx.doi.org/10.1016/j.msec.2004.02.005

Carpi, F., Migliore, A., Serra, G., et al., 2005. Helical dielectric elastomer actuators. Smart Materials \& Structures, 14(6):1210-1216.

Carpi, F., Bauer, S., de Rossi, D., 2010. Stretching dielectric elastomer performance. Science, 330(6012):1759-1761. http://dx.doi.org/10.1126/science.1194773

Chen, W.Q., 2014. The renaissance of continuum mechanics. Journal of Zhejiang University-SCIENCE A (Applied Physics \& Engineering), 15(4):231-240. http://dx.doi.org/10.1631/jzus.A1400079

Chen, W.Q., Dai, H.H., 2012. Waves in pre-stretched incompressible soft electroactive cylinders: exact solution. Acta Mechanica Solida Sinica, 25(5):530-541. http://dx.doi.org/10.1016/S0894-9166(12)60047-0

Chen, X., Mahadevan, L., Driks, A., et al., 2014. Bacillus spores as building blocks for stimuli-responsive materials and nanogenerators. Nature Nanotechnology, 9(2):137141. http://dx.doi.org/10.1038/nnano.2013.290

Davies, G.R., Yamwong, T., Voice, A.M., 2002. Electrostrictive response of an ideal polar rubber: comparison with experiment. Proc. SPIE 4695, Smart Structures and Materials 2002: Electroactive Polymer Actuators and Devices (EAPAD), 4695:111-119. http://dx.doi.org/10.1117/12.475155

Dollhofer, J., Chiche, A., Muralidharan, V., et al., 2004. Surface energy effects for cavity growth and nucleation in an incompressible neo-Hookean material-modeling and experiment. International Journal of Solids and Structures, 41(22-23):6111-6127. 
http://dx.doi.org/10.1016/j.ijsolstr.2004.04.041

Dorfmann, L., Ogden, R.W., 2014. Nonlinear Theory of Electroelastic and Magnetoelastic Interactions. Springer, USA, p.61-82.

Duncan, P.N., Nguyen, T.V., Hui, E.E., 2013. Pneumatic oscillator circuits for timing and control of integrated microfluidics. Proceedings of the National Academy of Sciences of the United States of America, 110(45): 18104-18109. http://dx.doi.org/10.1073/pnas.1310254110

Dünki, S.J., Ko, Y.S., Nüesch, F.A., et al., 2015. Self-repairable, high permittivity dielectric elastomers with large actuation strains at low electric fields. $A d$ vanced Functional Materials, 25(16):2467-2475. http://dx.doi.org/10.1002/adfm.201500077

Follador, M., Tramacere, F., Mazzolai, B., 2014. Dielectric elastomer actuators for octopus inspired suction cups. Bioinspiration \& Biomimetics, 9(4):046002. http://dx.doi.org/10.1088/1748-3182/9/4/046002

Foo, C.C., Cai, S., Koh, S.J.A., et al., 2012a. Model of dissipative dielectric elastomers. Journal of Applied Physics, 111(3):034102. http://dx.doi.org/10.1063/1.3680878

Foo, C.C., Koh, S.J.A., Keplinger, C., et al., 2012b. Performance of dissipative dielectric elastomer generators. Journal of Applied Physics, 111(9):094107. http://dx.doi.org/10.1063/1.4714557

Galantini, F., Bianchi, S., Castelvetro, V., et al., 2013. Functionalized carbon nanotubes as a filler for dielectric elastomer composites with improved actuation performance. Smart Materials and Structures, 22(5):055025.

Gent, A.N., 1996. A new constitutive relation for rubber. Rubber Chemistry and Technology, 69(1):59-61. http://dx.doi.org/10.5254/1.3538357

Godaba, H., Foo, C.C., Zhang, Z.Q., et al., 2014. Giant voltage-induced deformation of a dielectric elastomer under a constant pressure. Applied Physics Letters, 105(11): 112901 . http://dx.doi.org/10.1063/1.4895815

Goulbourne, N.C., 2011. A constitutive model of polyacrylate interpenetrating polymer networks for dielectric elastomers. International Journal of Solids and Structures, 48(7-8):1085-1091. http://dx.doi.org/10.1016/j.ijsolstr.2010.10.028

Goulbourne, N.C., Mockensturm, E.M., Frecker, M.I., 2007. Electro-elastomers: large deformation analysis of silicone membranes. International Journal of Solids and Structures, 44(9):2609-2626.

http://dx.doi.org/10.1016/j.ijsolstr.2006.08.015

Graf, C., Hitzbleck, J., Feller, T., et al., 2014. Dielectric elastomer-based energy harvesting: material, generator design, and optimization. Journal of Intelligent Material Systems and Structures, 25(8):951-966. http://dx.doi.org/10.1177/1045389X13502857

Ha, S.M., Yuan, W., Pei, Q.B., et al., 2006. Interpenetrating polymer networks for high-performance electroelastomer artificial muscles. Advanced Materials, 18(7):887-891. http://dx.doi.org/10.1002/adma.200502437
Haus, H., Matysek, M., Moessinger, H., et al., 2013. Modelling and characterization of dielectric elastomer stack actuators. Smart Materials and Structures, 22(10): 114009-114020.

He, T., Zhao, X., Suo, Z., 2009. Dielectric elastomer membranes undergoing inhomogeneous deformation. Journal of Applied Physics, 106(8):083522. http://dx.doi.org/10.1063/1.3253322

Hong, W., 2011. Modeling viscoelastic dielectrics. Journal of the Mechanics and Physics of Solids, 59(3):637-650. http://dx.doi.org/10.1016/j.jmps.2010.12.003

$\mathrm{Hu}$, Y., Suo, Z., 2012. Viscoelasticity and poroelasticity in elastomer gels. Acta Mechanica Solida Sinica, 25(5): 441-458. http://dx.doi.org/10.1016/S0894-9166(12)60039-1

Huang, J.S., Li, T.F., Foo, C.C., et al., 2012. Giant, voltageactuated deformation of a dielectric elastomer under dead load. Applied Physics Letters, 100(4):041911. http://dx.doi.org/10.1063/1.3680591

Huang, J.S., Shian, S., Suo, Z.G., et al., 2013. Maximizing the energy density of dielectric elastomer generators using equi-biaxial loading. Advanced Functional Materials, 23(40):5056-5061. http://dx.doi.org/10.1002/adfm.201300402

Huang, R., Suo, Z.G., 2012. Electromechanical phase transition in dielectric elastomers. Proceedings of the Royal Society A-Mathematical Physical and Engineering Sciences, 468(2140): 1014-1040. http://dx.doi.org/10.1098/rspa.2011.0452

Hui, T.H., Zhou, Z.L., Qian, J., et al., 2014. Volumetric deformation of live cells induced by pressure-activated cross-membrane ion transport. Physical Review Letters, 113(11): 118101 http://dx.doi.org/10.1103/PhysRevLett.113.118101

Jean-Mistral, C., Basrour, S., Chaillout, J.J., 2010. Modelling of dielectric polymers for energy scavenging applications. Smart Materials \& Structures, 19(10):105006-105016. http://dx.doi.org/10.1088/0964-1726/19/10/105006

Jiang, H.Y., Qian, J., Lin, Y., et al., 2015. Aggregation dynamics of molecular bonds between compliant materials. Soft Matter, 11(14):2812-2820. http://dx.doi.org/10.1039/C4SM02903H

Jordi, C., Michel, S., Fink, E., 2010. Fish-like propulsion of an airship with planar membrane dielectric elastomer actuators. Bioinspiration \& Biomimetics, 5(2):026007. http://dx.doi.org/10.1088/1748-3182/5/2/026007

Ju, L.N., Qian, J., Zhu, C., 2015. Transport regulation of two-dimensional receptor-ligand association. Biophysical Journal, 108(7):1773-1784. http://dx.doi.org/10.1016/j.bpj.2015.02.023

Jung, I., Xiao, J., Malyarchuk, V., et al., 2011. Dynamically tunable hemispherical electronic eye camera system with adjustable zoom capability. Proceedings of the National Academy of Sciences of the United States of America, 108(5):1788-1793. http://dx.doi.org/10.1073/pnas.1015440108

Kaal, W., Herold, S., 2013. Numerical investigations on dielectric stack actuators with perforated electrodes. Smart 
Materials and Structures, 22(10):104016.

Keplinger, C., Li, T.F., Baumgartner, R., et al., 2012. Harnessing snap-through instability in soft dielectrics to achieve giant voltage-triggered deformation. Soft Matter, 8(2):285-288.

http://dx.doi.org/10.1039/C1SM06736B

Khan, K.A., Wafai, H., El Sayed, T., 2013. A variational constitutive framework for the nonlinear viscoelastic response of a dielectric elastomer. Computational Mechanics, 52(2):345-360. http://dx.doi.org/10.1007/s00466-012-0815-6

Kofod, G., Kornbluh, R., Pelrine, R., et al., 2001. Actuation response of polyacrylate dielectric elastomers. Smart Structures and Materials 2001: Electroactive Polymer Actuators and Devices (EAPAD), 4329:141-147.

Kofod, G., Sommer-Larsen, P., Kronbluh, R., et al., 2003. Actuation response of polyacrylate dielectric elastomers. Journal of Intelligent Material Systems and Structures, 14(12):787-793. http://dx.doi.org/10.1177/104538903039260

Kofod, G., Wirges, W., Paajanen, M., et al., 2007. Energy minimization for self-organized structure formation and actuation. Applied Physics Letters, 90(8):081916. http://dx.doi.org/10.1063/1.2695785

Koh, S.J.A., Li, T.F., Zhou, J.X., et al., 2011. Mechanisms of large actuation strain in dielectric elastomers. Journal of Polymer Science Part B: Polymer Physics, 49(7): 504-515. http://dx.doi.org/10.1002/polb.22223

Kollosche, M., Zhu, J., Suo, Z.G., et al., 2012. Complex interplay of nonlinear processes in dielectric elastomers. Physical Review E, 85(5):976-986. http://dx.doi.org/10.1103/PhysRevE.85.051801

Kornbluh, R.D., Pelrine, R., Pei, Q.B., et al., 2000. Ultrahigh strain response of field-actuated elastomeric polymers. Proc. SPIE 3987, Smart Structures and Materials 2000: Electroactive Polymer Actuators and Devices (EAPAD), 3987:51-64. http://dx.doi.org/10.1117/12.387763

Kornbluh, R., Pelrine, R., Pei, Q.B., et al., 2002. Electroelastomers: applications of dielectric elastomer transducers for actuation, generation and smart structures. Proc. SPIE 4698, Smart Structures and Materials 2002: Industrial and Commercial Applications of Smart Structures Technologies, 4698:254-270. http://dx.doi.org/10.1117/12.475072

Kornbluh, R.D., Pelrine, R., Prahlad, H., et al., 2012. Dielectric elastomers: stretching the capabilities of energy harvesting. MRS Bulletin, 37(3):246-253. http://dx.doi.org/10.1557/mrs.2012.41

Kovacs, G., Duering, L., Michel, S., et al., 2009. Stacked dielectric elastomer actuator for tensile force transmission. Sensors and Actuators A: Physical, 155(2):299-307. http://dx.doi.org/10.1016/j.sna.2009.08.027

Leng, J., Liu, L., Liu, Y., et al., 2009. Electromechanical stability of dielectric elastomer. Applied Physics Letters, 94(21):211901. http://dx.doi.org/10.1063/1.3138153

Li, B., Zhou, J., Chen, H., 2011. Electromechanical stability in charge-controlled dielectric elastomer actuation. Applied Physics Letters, 99(24):244101. http://dx.doi.org/10.1063/1.3670048

Li, B., Chen, H., Qiang, J., et al., 2012. A model for conditional polarization of the actuation enhancement of a dielectric elastomer. Soft Matter, 8(2):311-317. http://dx.doi.org/10.1039/C1SM05847A

Li, B., Chen, H., Zhou, J., 2013. Electromechanical stability of dielectric elastomer composites with enhanced permittivity. Composites Part A: Applied Science and Manufacturing, 52(5):55-61. http://dx.doi.org/10.1016/j.compositesa.2012.11.013

Li, B., Zhang, J., Liu, L., et al., 2014. Modeling of dielectric elastomer as electromechanical resonator. Journal of Applied Physics, 116(12):124509. http://dx.doi.org/10.1063/1.4896584

Li, J.R., Liu, L.W., Liu, Y.J., et al., 2014. Dielectric elastomer bending actuator: experiment and theoretical analysis. Proc. SPIE 9056, Electroactive Polymer Actuators and Devices (EAPAD), No. 905639. http://dx.doi.org/10.1117/12.2045092

Li, T.F., Qu, S.X., Yang, W., 2012a. Energy harvesting of dielectric elastomer generators concerning inhomogeneous fields and viscoelastic deformation. Journal of Applied Physics, 112(3):034119. http://dx.doi.org/10.1063/1.4745049

Li, T.F., Qu, S.X., Keplinger, C., et al., 2012b. Modeling guided design of dielectric elastomer generators and actuators. Proc. SPIE 8340, Electroactive Polymer Actuators and Devices (EAPAD), No. 83401X. http://dx.doi.org/10.1117/12.915424

Li, T.F., Keplinger, C., Baumgartner, R., et al., 2013. Giant voltage-induced deformation in dielectric elastomers near the verge of snap-through instability. Journal of the Mechanics and Physics of Solids, 61(2):611-628. http://dx.doi.org/10.1016/j.jmps.2012.09.006

Li, T.F., Li, C., Mao, G.Y., et al., 2014a. Novel dielectric elastomer structures with electromechanical instability. Proc. SPIE 9056, Electroactive Polymer Actuators and Devices (EAPAD), No. 90560N. http://dx.doi.org/10.1117/12.2044959

Li, T.F., Zou, Z.N., Mao, G.Y., et al., 2014b. Electromechanical bistable behavior of a novel dielectric elastomer actuator. Journal of Applied Mechanics, 81(4):041019. http://dx.doi.org/10.1115/1.4025530

Liang, X., Cai, S., 2015. Shape bifurcation of a spherical dielectric elastomer balloon under the actions of internal pressure and electric voltage. Journal of Applied Mechanics, 82(10): 101002. http://dx.doi.org/10.1115/1.4030881

Liu, H., Zhang, L., Yang, D., et al., 2013. Mechanical, dielectric, and actuated strain of silicone elastomer filled with various types of $\mathrm{TiO}_{2}$. Soft Materials, 11(3): 363-370. http://dx.doi.org/10.1080/1539445X.2012.661821

Liu, L., Liu, Y., Leng, J.S., et al., 2011. Electromechanical stability of compressible dielectric elastomer actuators. 
Smart Materials and Structures, 20(11):115015.

Liu, L., Liu, Y., Yu, K., et al., 2014. Thermoelectromechanical stability of dielectric elastomers undergoing temperature variation. Mechanics of Materials, 72(5):33-45. http://dx.doi.org/10.1016/j.mechmat.2013.05.013

Liu, Y., Liu, L., Zhang, Z., et al., 2010. Analysis and manufacture of an energy harvester based on a MooneyRivlin-type dielectric elastomer. EPL (Europhysics Letters), 90(3):36004. http://dx.doi.org/10.1209/0295-5075/90/36004

Liu, Y., Gao, M., Mei, S., et al., 2013. Ultra-compliant liquid metal electrodes with in-plane self-healing capability for dielectric elastomer actuators. Applied Physics Letters, 103(6):064101. http://dx.doi.org/10.1063/1.4817977

Lu, T.Q., Huang, J.H., Jordi, C., et al., 2012. Dielectric elastomer actuators under equal-biaxial forces, uniaxial forces, and uniaxial constraint of stiff fibers. Soft Matter, 8(22):6167-6173. http://dx.doi.org/10.1039/c2sm25692d

Lu, T.Q., Cai, S.Q., Wang, H.M., et al., 2013. Computational model of deformable lenses actuated by dielectric elastomers. Journal of Applied Physics, 114(10):104104. http://dx.doi.org/10.1063/1.4821028

Lu, T.Q., Foo, C.C., Huang, J.H., et al., 2014. Highly deformable actuators made of dielectric elastomers clamped by rigid rings. Journal of Applied Physics, 115(18): 184105. http://dx.doi.org/10.1063/1.4876722

Maffli, L., Rosset, S., Shea, H.R., 2013. Zipping dielectric elastomer actuators: characterization, design and modeling. Smart Materials and Structures, 22(10):104013.

Magid, A., Law, D.J., 1985. Myofibrils bear most of the resting tension in frog skeletal-muscle. Science, 230(4731): 1280-1282. http://dx.doi.org/10.1126/science.4071053

Mao, G., Li, T., Zou, Z., et al., 2014. Prestretch effect on snap-through instability of short-length tubular elastomeric balloons under inflation. International Journal of Solids and Structures, 51(11-12):2109-2115. http://dx.doi.org/10.1016/j.ijsolstr.2014.02.013

Mao, G.Y., Huang, X.Q., Diab, M., et al., 2015. Nucleation and propagation of voltage-driven wrinkles in an inflated dielectric elastomer balloon. Soft Matter, 11(33):65696575 . http://dx.doi.org/10.1039/C5SM01102G

Meijer, K., Rosenthal, M., Full, R.J., 2001. Muscle-like actuators? A comparison between three electroactive polymers. Proc. SPIE 4329, Smart Structures and Materials 2001: Electroactive Polymer Actuators and Devices (EAPAD), 4329:7-15. http://dx.doi.org/10.1117/12.432649

Mockensturm, E.M., Goulbourne, N., 2006. Dynamic response of dielectric elastomers. International Journal of Non-Linear Mechanics, 41(3):388-395. http://dx.doi.org/10.1016/j.ijnonlinmec.2005.08.007

Morrow, D.A., Donahue, T.L.H., Odegard, G.M., et al., 2010. Transversely isotropic tensile material properties of skeletal muscle tissue. Journal of the Mechanical Be- havior of Biomedical Materials, 3(1):124-129.

http://dx.doi.org/10.1016/j.jmbbm.2009.03.004

Nguyen, C.H., Alici, G., Mutlu, R., 2014. A compliant translational mechanism based on dielectric elastomer actuators. Journal of Mechanical Design, 136(6):061009. http://dx.doi.org/10.1115/1.4027167

Niu, X.F., Leo, R., Chen, D., et al., 2013. Multilayer stack actuator made from new prestrain-free dielectric elastomers. Proc. SPIE 8687, Electroactive Polymer Actuators and Devices (EAPAD), No. 86871M. http://dx.doi.org/10.1117/12.2009561

O'Halloran, A., O'Malley, F., McHugh, P., 2008. A review on dielectric elastomer actuators, technology, applications, and challenges. Journal of Applied Physics, 104(7): 071101. http://dx.doi.org/10.1063/1.2981642

Park, H.S., Nguyen, T.D., 2013. Viscoelastic effects on electromechanical instabilities in dielectric elastomers. Soft Matter, 9(4):1031-1042. http://dx.doi.org/10.1039/C2SM27375F

Park, H.S., Suo, Z., Zhou, J., et al., 2012. A dynamic finite element method for inhomogeneous deformation and electromechanical instability of dielectric elastomer transducers. International Journal of Solids and Structures, 49(15-16):2187-2194. http://dx.doi.org/10.1016/j.ijsolstr.2012.04.031

Lochmatter, P., Kovacs, G., Wissler, M., 2007. Characterization of dielectric elastomer actuators based on a hyperelastic film model. Sensors and Actuators A: Physical, 135(2):748-757. http://dx.doi.org/10.1016/j.sna.2006.08.006

Pelrine, R., Kornbluh, R., Pei, Q.B., et al., 2000. High-speed electrically actuated elastomers with strain greater than 100\%. Science, 287(5454):836-839. http://dx.doi.org/10.1126/science.287.5454.836

Pelrine, R.E., Kornbluh, R.D., Joseph, J.P., 1998. Electrostriction of polymer dielectrics with compliant electrodes as a means of actuation. Sensors and Actuators A: Physical, 64(1):77-85. http://dx.doi.org/10.1016/S0924-4247(97)01657-9

Plante, J.S., Dubowsky, S., 2006. Large-scale failure modes of dielectric elastomer actuators. International Journal of Solids and Structures, 43(25-26):7727-7751. http://dx.doi.org/10.1016/j.ijsolstr.2006.03.026

Proulx, S., Chouinard, P., Bigue, J.P.L., et al., 2011. Design of a MRI-compatible dielectric elastomer powered jet valve. Proc. SPIE 7976, Electroactive Polymer Actuators and Devices (EAPAD), No. 79762C. http://dx.doi.org/10.1117/12.880670

Qian, J., Liu, H., Lin, Y., et al., 2013. A mechanochemical model of cell reorientation on substrates under cyclic stretch. PLoS ONE, 8(6):e65864. http://dx.doi.org/10.1371/journal.pone.0065864

Qu, S.X., Suo, Z.G., 2012. A finite element method for dielectric elastomer transducers. Acta Mechanica Solida Sinica, 25(5):459-466. http://dx.doi.org/10.1016/S0894-9166(12)60040-8

Qu, S.X., Li, K., Li, T.F., et al., 2012. Rate dependent 
stress-stretch relation of dielectric elastomers subjected to pure shear like loading and electric field. Acta Mechanica Solida Sinica, 25(5):542-549. http://dx.doi.org/10.1016/S0894-9166(12)60048-2

Reese, S., Govindjee, S., 1998. A theory of finite viscoelasticity and numerical aspects. International Journal of Solids and Structures, 35(26-27):3455-3482. http://dx.doi.org/10.1016/S0020-7683(97)00217-5

Reffaee, A.S.A., El Nashar, D.E., Abd-El-Messieh, S.L., et al., 2009. Electrical and mechanical properties of acrylonitrile rubber and linear low density polyethylene composites in the vicinity of the percolation threshold. Materials \& Design, 30(9):3760-3769. http://dx.doi.org/10.1016/j.matdes.2009.02.001

Rosset, S., Niklaus, M., Dubois, P., et al., 2009. Metal ion implantation for the fabrication of stretchable electrodes on elastomers. Advanced Functional Materials, 19(3): 470-478.

http://dx.doi.org/10.1002/adfm.200801218

Sarangapani, K.K., Qian, J., Chen, W.Q., et al., 2011. Regulation of catch bonds by rate of force application. Journal of Biological Chemistry, 286(37):32749-32761. http://dx.doi.org/10.1074/jbc.M111.240044

Seki, M., Sato, K., Haga, Y., et al., 1995. Electrical-properties of acrylic elastomer- $\mathrm{LiClO}_{4}$ complex as a polymeric solid-electrolyte. Macromolecular Chemistry and Physics, 196(6): 1813-1820. http://dx.doi.org/10.1002/macp.1995.021960602

Shankar, R., Ghosh, T.K., Spontak, R.J., 2007. Dielectric elastomers as next-generation polymeric actuators. Soft Matter, 3(9):1116-1129. http://dx.doi.org/10.1039/b705737g

Sheng, J., Chen, H., Qiang, J., et al., 2012. Thermal, mechanical, and dielectric properties of a dielectric elastomer for actuator applications. Journal of Macromolecular Science, Part B: Physics, 51(10):2093-2104. http://dx.doi.org/10.1080/00222348.2012.659617

Sheng, J., Chen, H., Liu, L., et al., 2013. Dynamic electromechanical performance of viscoelastic dielectric elastomers. Journal of Applied Physics, 114(13):134101. http://dx.doi.org/10.1063/1.4823861

Suo, Z.G., 2010. Theory of dielectric elastomers. Acta Mechanica Solida Sinica, 23(6):549-578. http://dx.doi.org/10.1016/S0894-9166(11)60004-9

Suo, Z.G., 2012. Mechanics of stretchable electronics and soft machines. MRS Bulletin, 37(3):218-225. http://dx.doi.org/10.1557/mrs.2012.32

Suo, Z.G., Zhu, J., 2009. Dielectric elastomers of interpenetrating networks. Applied Physics Letters, 95(23):232909. http://dx.doi.org/10.1063/1.3272685

Suo, Z.G., Zhao, X., Greene, W.H., 2008. A nonlinear field theory of deformable dielectrics. Journal of the Mechanics and Physics of Solids, 56(2):467-486. http://dx.doi.org/10.1016/j.jmps.2007.05.021

Tian, L., Tevet-Deree, L., deBotton, G., et al., 2012. Dielectric elastomer composites. Journal of the Mechanics and
Physics of Solids, 60(1):181-198.

http://dx.doi.org/10.1016/j.jmps.2011.08.005

Tian, M., Yan, B., Yao, Y., et al., 2014. Largely improved actuation strain at low electric field of dielectric elastomer by combining disrupting hydrogen bonds with ionic conductivity. Journal of Materials Chemistry C, 2(39): 8388-8397. http://dx.doi.org/10.1039/C4TC01140F

Tutcuoglu, A., Majidi, C., 2014. Energy harvesting with stacked dielectric elastomer transducers: nonlinear theory, optimization, and linearized scaling law. Applied Physics Letters, 105(24):214905.

http://dx.doi.org/10.1063/1.4904473

van Loocke, M., Lyons, C.G., Simms, C.K., 2008. Viscoelastic properties of passive skeletal muscle in compression: stress-relaxation behaviour and constitutive modelling. Journal of Biomechanics, 41(7):1555-1566. http://dx.doi.org/10.1016/j.jbiomech.2008.02.007

van Loocke, M., Simms, C.K., Lyons, C.G., 2009. Viscoelastic properties of passive skeletal muscle in compressioncyclic behaviour. Journal of Biomechanics, 42(8): 1038-1048. http://dx.doi.org/10.1016/j.jbiomech.2009.02.022

Vertechy, R., Frisoli, A., Bergamasco, M., et al., 2012. Modeling and experimental validation of buckling dielectric elastomer actuators. Smart Materials and Structures, 21(9):094005.

Vu-Cong, T., Jean-Mistral, C., Sylvestre, A., 2013. New operating limits for applications with electroactive elastomer: effect of the drift of the dielectric permittivity and the electrical breakdown. Proc. SPIE 8687, Electroactive Polymer Actuators and Devices (EAPAD), No. 86871S. http://dx.doi.org/10.1117/12.2007698

Wang, H., Cai, S., Carpi, F., et al., 2012. Computational model of hydrostatically coupled dielectric elastomer actuators. Journal of Applied Mechanics, 79(3):031008. http://dx.doi.org/10.1115/1.4005885

Wang, H., Lei, M., Cai, S., 2013. Viscoelastic deformation of a dielectric elastomer membrane subject to electromechanical loads. Journal of Applied Physics, 113(21): 213508 . http://dx.doi.org/10.1063/1.4807911

Wang, J., Nguyen, T.D., Park, H.S., 2014. Electrostatically driven creep in viscoelastic dielectric elastomers. Journal of Applied Mechanics, 81(5): 1847-1858. http://dx.doi.org/10.1115/1.4025999

Wang, Y., Zhou, J.X., Wu, X.H., et al., 2013. Energy diagrams of dielectric elastomer generators under different types of deformation. Chinese Physics Letters, 30(6):66103. http://dx.doi.org/10.1088/0256-307X/30/6/066103

Wang, Y., Chen, B.H., Bai, Y.Y., et al., 2014a. Actuating dielectric elastomers in pure shear deformation by elastomeric conductors. Applied Physics Letters, 104(6): 064101.

http://dx.doi.org/10.1063/1.4864402

Wang, Y., Zhou, J.W., Sun, W.J., et al., 2014b. Mechanics of 
dielectric elastomer-activated deformable transmission grating. Smart Materials and Structures, 23(9):095010.

Wang, Y.Q., Xue, H.H., Chen, H.L., et al., 2013. A dynamic visco-hyperelastic model of dielectric elastomers and their energy dissipation characteristics. Applied Physics A-Materials Science \& Processing, 112(2):339-347. http://dx.doi.org/10.1007/s00339-013-7740-1

Wissler, M., Mazza, E., 2005. Modeling and simulation of dielectric elastomer actuators. Smart Materials \& Structures, 14(6):1396-1402. http://dx.doi.org/10.1088/0964-1726/14/6/032

Wu, X., Sun, W., Li, B., et al., 2012. Homogeneous large deformation analysis of a dielectric elastomer peristaltic actuator. Science China-Technological Sciences, 55(2): 537-541. http://dx.doi.org/10.1007/s11431-011-4645-0

Zhang, C., Chen, H.L., Liu, L., et al., 2015. Modelling and characterization of inflated dielectric elastomer actuators with tubular configuration. Journal of Physics D: Applied Physics, 48(24):245502. http://dx.doi.org/10.1088/0022-3727/48/24/245502

Zhang, J., Chen, H., Li, B., 2014a. A method of tuning viscoelastic creep in charge-controlled dielectric elastomer actuation. EPL (Europhysics Letters), 108(5):57002. http://dx.doi.org/10.1209/0295-5075/108/57002

Zhang, J., Chen, H., Sheng, J., et al., 2014b. Dynamic performance of dissipative dielectric elastomers under alternating mechanical load. Applied Physics A-Materials Science \& Processing, 116(1):59-67. http://dx.doi.org/10.1007/s00339-013-8092-6

Zhang, W.L., Lin, Y., Qian, J., et al., 2013. Tuning molecular adhesion via material anisotropy. Advanced Functional Materials, 23(37):4729-4738. http://dx.doi.org/10.1002/adfm.201300069

Zhang, X.Q., Lowe, C., Wissler, M., et al., 2005. Dielectric elastomers in actuator technology. Advanced Engineering Materials, 7(5):361-367. http://dx.doi.org/10.1002/adem.200500066

Zhang, Z., Liu, L.W., Liu, Y.J., et al., 2012. Effect of mechanical force field on the electromechanical stability of dielectric elastomers. Science China-Physics Mechanics \& Astronomy, 55(1):94-101. http://dx.doi.org/10.1007/s11433-011-4537-0

Zhao, X.H., Suo, Z.G., 2007. Method to analyze electromechanical stability of dielectric elastomers. Applied Physics Letters, 91(6):061921. http://dx.doi.org/10.1063/1.2768641

Zhao, X.H., Wang, Q.M., 2014. Harnessing large deformation and instabilities of soft dielectrics: theory, experiment, and application. Applied Physics Reviews, 1(2):021304. http://dx.doi.org/10.1063/1.4871696

Zhao, X.H., Hong, W., Suo, Z.G., 2007. Electromechanical hysteresis and coexistent states in dielectric elastomers. Physical Review B, 76(13):134113. http://dx.doi.org/10.1103/PhysRevB.76.134113
Zhao, X.H., Koh, S.J.A., Suo, Z.G., 2011. Nonequilibrium thermodynamics of dielectric elastomers. International Journal of Applied Mechanics, 3(2):203-217. http://dx.doi.org/10.1142/S1758825111000944

Zhou, J.Y., Jiang, L.Y., Khayat, R.E., 2013. Failure analysis of a dielectric elastomer plate actuator considering boundary constraints. Journal of Intelligent Material Systems and Structures, 24(14):1667-1674. http://dx.doi.org/10.1177/1045389X13483025

Zhou, J.Y., Jiang, L.Y., Khayat, R.E., 2014a. Electromechanical response and failure modes of a dielectric elastomer tube actuator with boundary constraints. Smart Materials and Structures, 23(4):045028.

Zhou, J.Y., Jiang, L.Y., Khayat, R.E., 2014b. Viscoelastic effects on frequency tuning of a dielectric elastomer membrane resonator. Journal of Applied Physics, 115(12): 24106. http://dx.doi.org/10.1063/1.4869666

Zhu, J., Stoyanov, H., Kofod, G., et al., 2010. Large deformation and electromechanical instability of a dielectric elastomer tube actuator. Journal of Applied Physics, 108(7):074113. http://dx.doi.org/10.1063/1.3490186

Zou, Z., Li, T., Qu, S., et al., 2014. Active shape control and phase coexistence of dielectric elastomer membrane with patterned electrodes. Journal of Applied Mechanics, 81(3):031016.

http://dx.doi.org/10.1115/1.4025416

\section{中文概要}

\section{题 目: 介电高弹体的材料、结构和器件力学}

目 的: 介电高弹体是典型电敏性材料, 在外加电场的作 用下会产生大的变形, 这一特点使其成为人工肌 肉致动的理想材料, 近年来引起研究者的广泛关 注。本文着重介绍介电高弹体的基本力学理论和 方法, 旨在为相关材料、结构和器件的设计提供 参考, 也有助于不同专业背景的研究者了解并开 展介电高弹体的相关研究。

概 要: 本文介绍了近年来关于介电高弹体力电耦合问题 的一些理论和数值研究, 重点包括力电耦合的控 制方程、材料本构关系、粘弹性响应、力电失稳 以及致动器设计等方面。文中讨论了基于非平衡 热动力学的介电高弹体力学模型处理复杂构型 或与时间相关变形时常被采用的数值方法, 优化 介电高弹体致动极限的力学设计, 以及介电高弹 体力电响应在典型致动器中的应用。

关键词: 人工肌肉; 智能材料; 介电高弹体; 力电耦合; 本构关系; 粘弹性; 力电失稳; 致动 